\title{
Measurement Differences, Faults and Instabilities in Intelligent Energy Systems - Part 2: Fault and Instability Prediction in Overhead High-Voltage Broadband over Power Lines Networks by Applying Fault and Instability Identification Methodology (FIIM)
}

\author{
Athanasios G. Lazaropoulos* \\ School of Electrical and Computer Engineering, National Technical University of Athens, \\ 9 IroonPolytechniou Street, Zografou, GR 15780 Greece
}

Received August 26, 2016; Accepted September 23, 2016; Published October 3, 2016

\begin{abstract}
This companion paper of [1] focuses on the prediction of various faults and instabilities that may occur during the operation of the transmission power grid when overhead high-voltage broadband over power lines (OV HV BPL) networks are deployed across it. Having already been identified the theoretical OV HV BPL transfer function for a given OV HV BPL network [1], the faults and instabilities of the transmission power grid are first differentiated from the measurement differences, which can occur during the determination of an OV HV BPL transfer function, and, then, are identified by applying the best L1 Piecewise Monotonic data Approximation (best L1PMA) to the measured OV HV BPL transfer function. When faults and instabilities are detected, a warning is issued.

The contribution of this paper is triple. First, the Topology Identification Methodology (TIM) of [1] is here extended to the proposed Fault and Instability Identification Methodology (FIIM) so that faults and instabilities across the transmission power grid can be identified. Also, the curve similarity performance percentage metric (CSPpM) that acts as the accompanying performance metric of FIIM is introduced. Second, the impact of various fault and instability conditions on the OV HV BPL transfer functions is demonstrated. Third, the fault and instability prediction procedure by applying the FIIM is first reported.
\end{abstract}

Keywords: Smart Grid; Intelligent Energy Systems; Broadband over Power Lines (BPL) networks; Power Line Communications (PLC); Faults; Fault Analysis; Transmission Power Grids

\section{Introduction}

The deployment of broadband over power lines (BPL) networks across the vintage overhead high-voltage (OV HV) power grid can transform it into a modern IP-based communications network with a great number of smart grid applications [1]. Among the available smart grid applications, this companion paper focuses on the transmission power grid protection that is a critical matter for both operational and safety reasons [2]-[5]. 
In fact, the development of an efficient protection scheme, which can ensure the adequate operation of the power grid as well as the protection of its equipment, has attracted the academic interest for many years. The protection scheme proposals have mainly been based on traditional circuit analysis solutions [6], artificial intelligence methods [7]-[10], wavelet transformations [11]-[15] and narrowband power line communications applications [12], [13], [16]. Only recently, the broadband potential of power grid has been recognized and exploited as a formidable basis on which the protection capability of the power grid is further enhanced [2], [17]-[21].

In this companion paper, the Fault and Instability Identification Methodology (FIIM) is proposed that exploits the well-validated knowledge concerning: (i) the BPL network operation across transmission and distribution power grids (i.e., hybrid method of [22]-[36]); (ii) the anti-fault operation of best L1PMA [1], [37]; and (iii) the Topology Identification Methodology (TIM) of [1]. More analytically, a number of practical reasons and "real-life" conditions may create measurement differences between experimental and theoretical results during the transfer function determination of OV HV BPL networks. By adopting best L1PMA, which is theoretically presented and experimentally verified in various systems including transmission and distribution BPL networks [1], [37]-[44], an OV HV BPL network topology can be identified despite the fact that measurement differences occur during the determination of its measured OV HV BPL transfer function. However, various serious faults and instabilities can occur across the transmission power grid and create significant fault conditions whose nature differ from the aforementioned "innocent" measurement differences (e.g., line faults and instabilities). By applying FIIM, these fault conditions are first differentiated from the measurement differences and then are identified. In fact, the contribution of the FIIM in comparison with the traditional one is that it can surely identify fault and instability conditions without creating a fault alarm situation despite the fact that measurement differences occur. From the moment that a fault or instability is identified, the personnel, who is monitoring the transmission power grid, is warned so as to restore the power grid to default settings.

The rest of this companion paper is organized as follows: In Sec. II, the faults and instabilities, which are examined in this paper, are detailed with reference to indicative topologies and the hybrid-method. In Sec. III, a presentation of the FIIM and its appropriate curve similarity performance percentage metric (CSPpM) are given. Sec.IV discusses the simulations of various transmission BPL networks intending to mark out the efficiency of FIIM to issue warnings due to serious faults and instabilities of the transmission intelligent energy system. Sec.V concludes this paper.

\section{Faults and Instabilities in Transmission Power Grids}

\subsection{Indicative OV HV BPL Topologies}

In accordance with [1], [22]-[35], [45]-[48] and with reference to Fig. 1(a), average path lengths of the order of $25 \mathrm{~km}$ are considered in OV HV BPL topologies. Apart from the three indicative OV HV BPL topologies of [1], another one that describes the OV HV BPL signal propagation and transmission in more aggravated environments such those of urban areas is added. Hence, the following four indicative OV HV BPL topologies, concerning end-to-end connections of average path lengths, are examined, as follows: 
1. A typical urban topology (OV HV urban case) with $N=3$ branches $\left(L_{1}=1 \mathrm{~km}, L_{2}=12 \mathrm{~km}, L_{3}=8 \mathrm{~km}, L_{4}=4 \mathrm{~km}, L_{\mathrm{b} 1}=24 \mathrm{~km}, L_{\mathrm{b} 2}=2 \mathrm{~km}, L_{\mathrm{b} 3}=7 \mathrm{~km}\right)$.

2. A typical suburban topology (OV HV suburban case) with $N=2$ branches $\left(L_{1}=9 \mathrm{~km}\right.$, $\left.L_{2}=13 \mathrm{~km}, L_{3}=3 \mathrm{~km}, L_{\mathrm{b} 1}=17 \mathrm{~km}, L_{\mathrm{b} 2}=13 \mathrm{~km}\right)$.

3. A typical rural topology (OV HV rural case) with only $N=1$ branch $\left(L_{1}=4 \mathrm{~km}, L_{2}=21 \mathrm{~km}, L_{\mathrm{b} 1}=24 \mathrm{~km}\right)$.

4. The "LOS" transmission along the same end-to-end distance $L=L_{1}+\ldots+L_{N+1}=25 \mathrm{~km}$ when no branches are encountered. This topology corresponds to Line of Sight transmission in wireless channels.

The four indicative OV HV BPL topologies are going to be used so that the accuracy of the proposed FIIM is evaluated in Sec.IV.

The assumptions for the circuital parameters of OV HV BPL topologies, which are concerned in this companion paper, are the same with [1], namely: (i) The branch lines are assumed identical to the transmission lines; (ii) The interconnections between the transmission and branch conductors of the lines are fully activated; (iii) The transmitting and the receiving ends are assumed matched to the characteristic impedance of the modal channels; and (iv) The branch terminations are assumed to be open circuits.

\subsection{Modeling of Faults and Instabilities in OV HV BPL Topologies}

As already been mentioned in [1], a set of practical reasons and "real-life" conditions can create significant differences between measurements and theoretical results during the transfer function determination of BPL networks. The reasons for these measurement differences have been grouped into six categories [1], [37], [49]-[51]. According to [1], [37], the total occurred fault due to the six measurement difference categories can be assumed to follow either continuous uniform distribution (CUD) with minimum value - $a_{\text {CUD }}$ and maximum value $a_{\text {CUD }}$ or normal distribution (ND) with mean $m_{\mathrm{ND}}$ and standard deviation $s_{\mathrm{ND}}$.

Apart from the aforementioned "innocent" measurement differences, which can be anyway satisfactorily mitigated by the TIM of [1], various serious problematic conditions across the transmission power grid can occur whose nature differs from this of the aforementioned measurement differences. In fact, these problematic conditions cause permanent damage to the transmission power grid, do not follow any error distribution and their impact on the determination of OV HV BPL transfer functions totally change the form of the result. In this paper, the problematic conditions are divided into two categories, namely: 


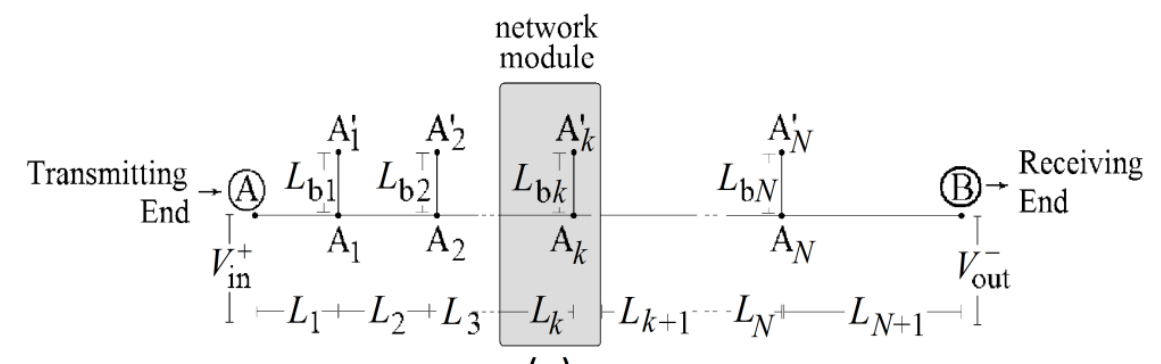

(a)

\section{Faults}

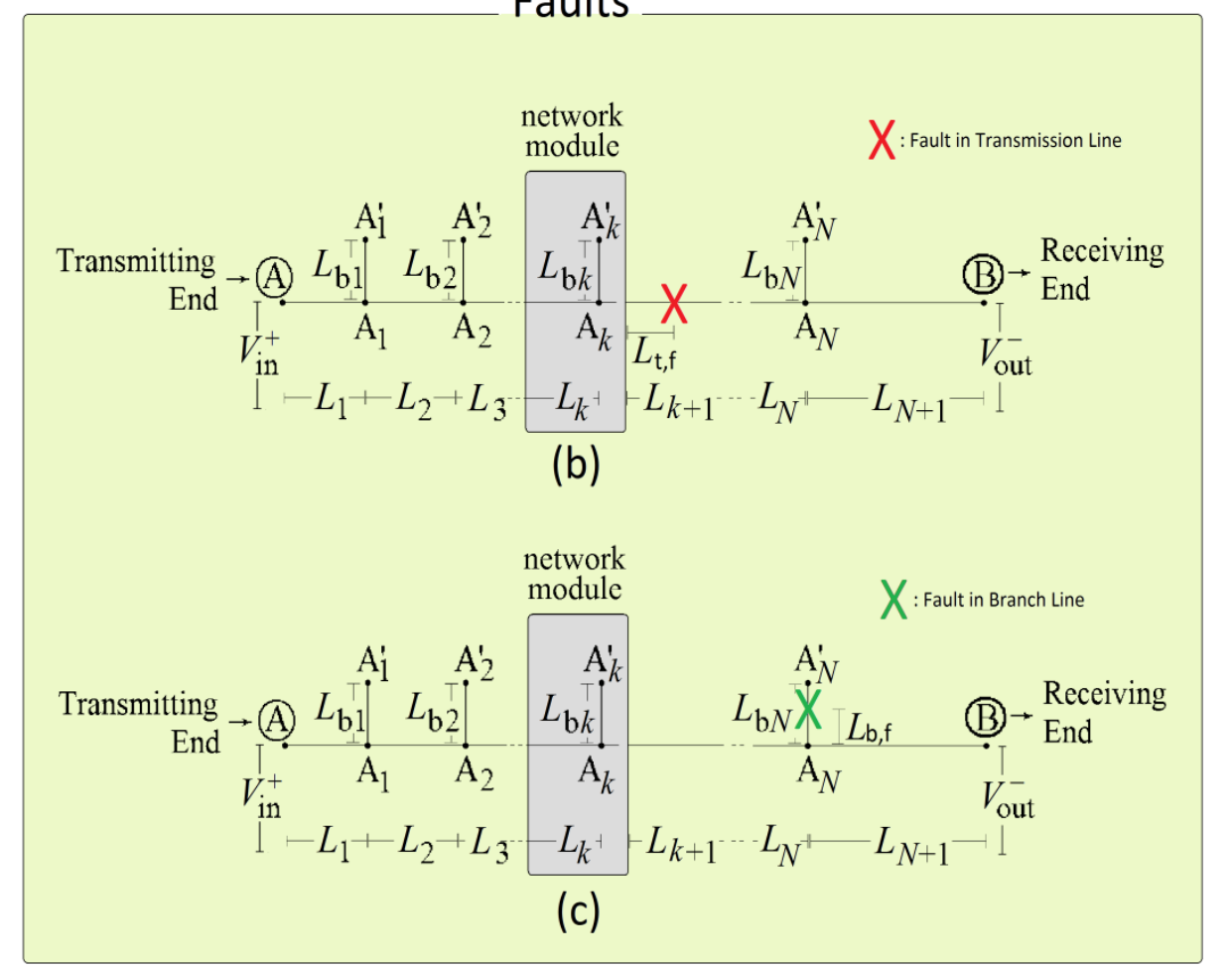

\section{Instabilities}

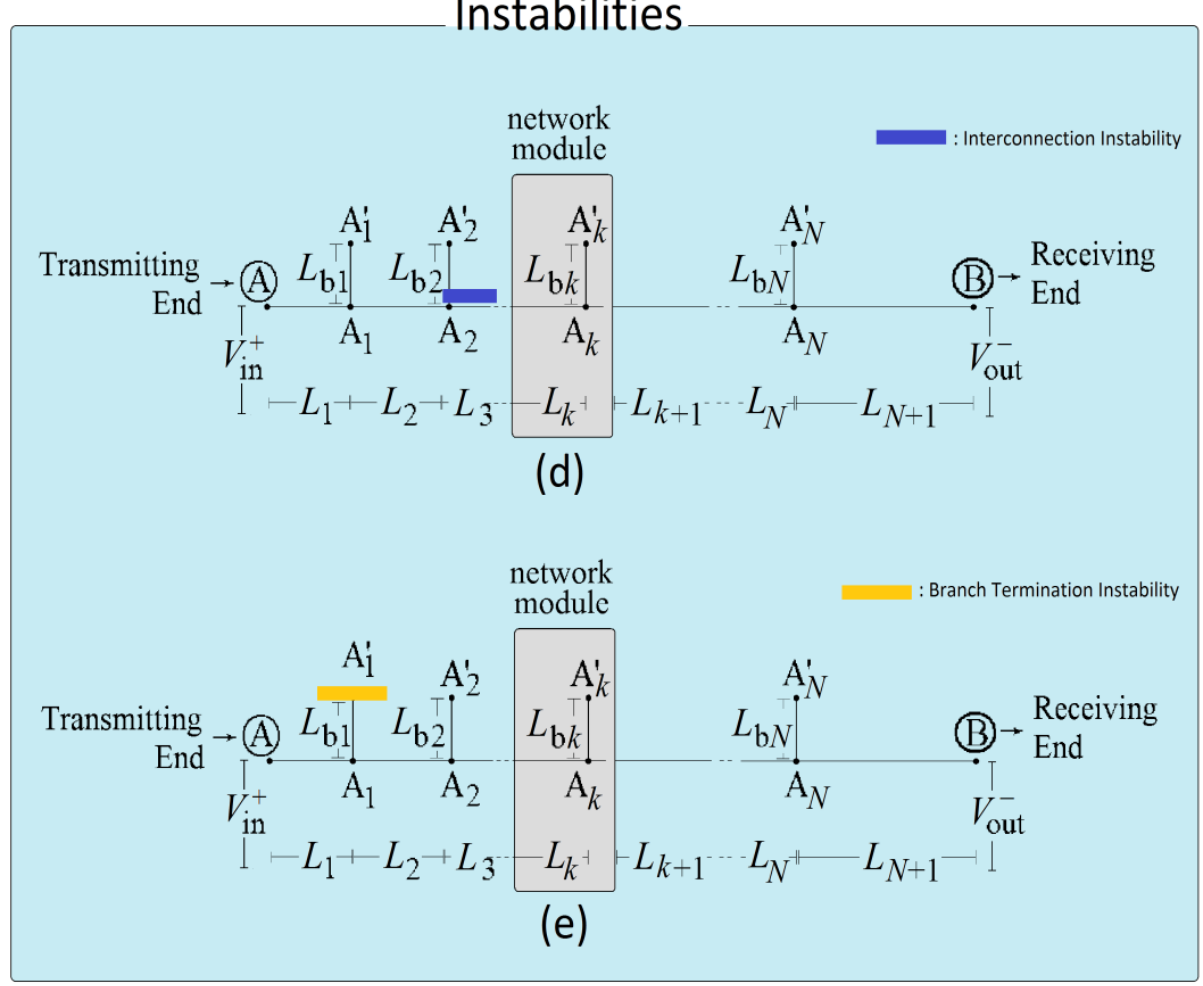

Figure 1. (a) General OV HV BPL topology [1]. (b, c) Faults in OV HV BPL topologies. (d, e) Instabilities in OV HV BPL topologies. 
- Faults: This category describes all the interruptions that can occur in the lines of a transmission power grid. There are two subcategories of line interruptions that are examined in this paper:

- Fault in Transmission Line: This fault subcategory describes the condition where a transmission line is interrupted due to physical or human reasons. With reference to Fig. 1(b), let the transmission line be broken at the position $\sum_{i=1}^{k} L_{k}+L_{\mathrm{t}, \mathrm{f}}$ from the transmitting end. This incident has as a result the communications failure between the transmitting and receiving end. Since all the other BPL network settings are well-verified, the personnel that is responsible for the transmission power grid operation is immediately warned by the FIIM with the reason of a fault in transmission line. The methodology of the exact fault position has already been presented in [2], [17]-[20]. Anyway, the localization of the faults and instabilities is outside of the scope of this paper and is not analyzed further.

- Fault in Branch Line: Similarly to faults in transmission lines, this fault subcategory describes the condition where a branch line is interrupted. With reference to Fig. 1(c), let the branch $N$ be broken at the position $L_{\mathrm{b}, \mathrm{f}}$ from the branching interconnection $\mathrm{A}_{N}$ with the transmission line. In contrast with faults in transmission lines, this problematic condition does not critically influence the communication between the transmitting and receiving end, nevertheless, it may create permanent undetectable failure and severely degrade the BPL network performance. However, the interruption of the $N$ branch (i.e., open circuit) transfers the termination from $L_{\mathrm{b} N}$ to $L_{\mathrm{b}, \mathrm{f}}$, thus, converting the examined $N$-branch $\mathrm{OV}$ HV BPL topology into a new $N$-branch OV HV BPL topology. Even if measurement differences occur, FIIM recognizes this fault condition and warns the responsible personnel. Simulation results concerning faults in branch lines and their identification by FIIM are presented in Sec.IVD.

- Instabilities: This category describes all the failures that can occur in the equipment across the transmission power grid. There are two subcategories of equipment failures that are examined in this paper:

- Instability in Branch Interconnections: One assumption of the hybrid method definition is that the interconnections between the transmission and branch lines are fully activated. However, these connectors may present faults having as a result the partial or total interruption of the branch line. With reference to Fig. 1(d), the total interruption of the second branch at the point $\mathrm{A}_{2}$ cancels the presence of this branch, thus, converting the examined $N$-branch OV HV BPL topology into a $(N-1)$-branch OV HV BPL topology. Depending on the termination of this branch (i.e., transformer $\mathrm{HV} / \mathrm{MV}$, load or source), significant instability during the operation of the transmission power grid can occur. FIIM can recognize this instability condition and warn the responsible personnel. Simulation results concerning instabilities in branch interconnections and their identification by FIIM are presented in Sec.IVE. 
- Instability in Branch Terminations: Again, one assumption of the fine hybrid method operation is that the branch terminations are assumed to be open circuits. This is due to the fact that HV/MV transformers are connected to the branch terminations, which are treated as open circuit terminations when high-frequency signals are considered. Physical stress on transformer windings, overloads, thermal aging of winding insulation, mechanical shake of windings and overflow or tank rupture are among the main causes of faults in HV/MV transformers. Since HV/MV transformers are critical pieces of equipment during the operation of power systems, their failure can create significant instabilities during their operation. With reference to Fig. 1(e), let the branch termination of first branch stop to act as open circuit termination. In that case, the branch termination fluctuates and so does the performance of transmission power grid. Again, FIIM can recognize this instability condition and warn the responsible personnel. Simulation results concerning instabilities in branch terminations and their identification by FIIM are presented in Sec.IVF.

\section{FIIM and CSPpM}

\subsection{Implementation Details}

In accordance with [1], a set of practical reasons and "real-life" conditions create significant differences between experimental measurements and theoretical results during the transfer function determination of BPL networks. The reasons for these measurement differences have been grouped into six categories. Then, the measured OV HV coupling transfer function $\overline{H^{\mathrm{WtG}}}\{\cdot\}$ is determined by

$$
\overline{H^{\mathrm{WtG}}}\left(f_{i}\right)=H^{\mathrm{WtG}}\left(f_{i}\right)+e\left(f_{i}\right)
$$

where $f_{i}$ denotes the measurement frequency, $H^{\mathrm{WtG}}\{\cdot\}$ is the theoretical OV HV coupling transfer function and $e\left(f_{i}\right)$ synopsizes the total measurement difference due to the aforementioned six categories. The measurement frequencies $f_{i}, i=1, \ldots, u$, the measured $\mathrm{OV} \mathrm{HV}$ coupling transfer functions $\overline{H^{\mathrm{WtG}}}\left(f_{i}\right), i=1, \ldots, u$ and the theoretical $\mathrm{OV} \mathrm{HV}$ coupling transfer functions $H^{\mathrm{WtG}}\left(f_{i}\right), i=1, \ldots, u$ can be treated as elements of $\mathbf{f}, \overline{\mathbf{H}^{\mathrm{WtG}}}$ and $\mathbf{H}^{\mathrm{WtG}}$, respectively, where

$$
\begin{aligned}
\mathbf{f}=\left[\begin{array}{lllll}
f_{1} & \cdots & f_{i} & \cdots & f_{u}
\end{array}\right]^{\mathrm{T}}, i=1, \ldots, u \\
\overline{\mathbf{H}^{\mathrm{WtG}}} \equiv \overline{\mathbf{H}^{\mathrm{WtG}}}(\mathbf{f})=\left[\begin{array}{lllll}
\overline{H^{\mathrm{WtG}}}\left(f_{1}\right) & \cdots & \overline{H^{\mathrm{WtG}}}\left(f_{i}\right) & \cdots & \overline{H^{\mathrm{WtG}}}\left(f_{u}\right)
\end{array}\right]^{\mathrm{T}}, i=1, \ldots, u \\
\mathbf{H}^{\mathrm{WtG}} \equiv \mathbf{H}^{\mathrm{WtG}}(\mathbf{f})=\left[\begin{array}{lllll}
H^{\mathrm{WtG}}\left(f_{1}\right) & \cdots & H^{\mathrm{WtG}}\left(f_{i}\right) & \cdots & H^{\mathrm{WtG}}\left(f_{u}\right)
\end{array}\right]^{\mathrm{T}}, i=1, \ldots, u
\end{aligned}
$$

As the application of best L1PMA is concerned, best L1PMA and its corresponding curve similarity performance metric (CSPM) achieve to reveal the OV HV BPL topology even though measurement differences of various distributions and magnitudes have been applied [1]. In this paper, each indicative OV HV BPL topology of Sec.IIA is accompanied with a number of approximated measured OV HV BPL coupling transfer function column vector $\overline{\overline{\mathbf{H}_{\text {meas }}^{\mathrm{WtG}}}}\left(\mathbf{f}, k_{\text {sect }}\right)$ and approximated theoretical OV HV BPL 
coupling transfer function column vector $\overline{\overline{\mathbf{H}_{\text {theor }}^{\mathrm{WtG}}}}\left(\mathbf{f}, k_{\text {sect }}\right)$ that come from the application of the best L1PMA for different monotonic sections $k_{\text {sect }}$. Note that monotonic sections may range from 1 to $k_{\text {sect,max }}$ where $k_{\text {sect,max }}$ is the maximum number of monotonic sections considered in this paper. Hence, for each indicative OV HV BPL topology, the total $C S P M$ tot is determined from

$$
C S P M_{\text {tot }} \equiv \sum_{k_{\text {sect }}=1}^{k_{\text {sect } \max }} \operatorname{CSPM} M_{k_{\text {sect }}}
$$

where

$$
C S P M_{k_{\text {sect }}} \equiv C S P M_{k_{\text {sect }}}\left(\overline{\mathbf{H}^{\mathrm{WtG}}}, \mathbf{H}^{\mathrm{WtG}}, k_{\text {sect }}\right)=\sum_{i=1}^{u} \mid \overline{\overline{\mathbf{H}_{\text {meas }}^{\mathrm{WtG}}}}\left(f_{i}, k_{\text {sect }}\right)-\overline{\overline{\mathbf{H}_{\text {theor }}^{\mathrm{WtG}}}}\left(f_{i}, k_{\text {sect }}\right)
$$

denotes the sum of the absolute errors between the best L1PMA measurement approximation and best L1PMA theory approximation for given monotonic section.

On the basis of eq.(5) and eq.(6), the proposed curve similarity performance percentage metric (CSPpM), which acts as the accompanying performance metric of FIIM, is here determined from

$$
C S P p M \equiv 100 \% \cdot \frac{\sum_{k_{\text {sect }}=1}^{k_{\text {sect, } \max }} \sum_{i=1}^{u} \frac{\left|\overline{\overline{\mathbf{H}_{\text {meas }}^{\mathrm{WtG}}}}\left(f_{i}, k_{\text {sect }}\right)-\overline{\overline{\mathbf{H}_{\text {theor }}^{\mathrm{WtG}}}}\left(f_{i}, k_{\text {sect }}\right)\right|}{\left|\overline{\overline{\mathbf{H}_{\text {theor }}^{\mathrm{WtG}}}}\left(f_{i}, k_{\text {sect }}\right)\right|}}{k_{\text {sect, } \max } \times u}
$$

Actually, CSPpM is a percentage metric that presents a specific pattern for an OV HV BPL topology. Hence, on the basis of TIM of [1], CSPpM may easily be retrieved during the normal operation of the transmission power grid for given OV HV BPL topology. As it is verified in Sec.IVB, this pattern stability allows CSPpM to act as health metric for an OV HV BPL topology.

In accordance with Sec.IIB, if a fault or instability occurs across the examined original OV HV BPL topology then a modified OV HV BPL topology should be examined. In that case, the measured OV HV coupling transfer function $\overline{H^{\mathrm{WtG}^{*}}}\{\cdot\}$ of the modified topology is determined by

$$
\overline{H^{\mathrm{WtG}}}\left(f_{i}\right)=H^{\mathrm{WtG}}\left(f_{i}\right)+e^{*}\left(f_{i}\right)
$$

where $H^{\mathrm{WtG}}\{\cdot\}$ is the theoretical OV HV coupling transfer function of the modified topology and $e^{*}\left(f_{i}\right)$ synopsizes the new total measurement difference due to the aforementioned six categories. Similarly to the original OV HV BPL topology, the CSPpM of the modified OV HV BPL topology is given from

$$
\operatorname{CSPPM} M^{*} \equiv 100 \% \cdot \frac{\sum_{k_{\text {sect }}=1}^{k_{\text {sect,max }}} \sum_{i=1}^{u} \frac{\left|\overline{\mathbf{H}_{\text {meas }}^{\mathrm{WtG}^{*}}}\left(f_{i}, k_{\text {sect }}\right)-\overline{\overline{\mathbf{H}_{\text {theor }}^{\mathrm{WtG}}}}\left(f_{i}, k_{\text {sect }}\right)\right|}{\left|\overline{\overline{\mathbf{H}_{\text {theor }}^{\mathrm{WtG}}}}\left(f_{i}, k_{\mathrm{sect}}\right)\right|}}{k_{\text {sect, } \max } \times u}
$$

where $\overline{\overline{\mathbf{H}_{\text {meas }}^{\mathrm{Wt} \mathrm{G}^{*}}}}\left(\mathbf{f}, k_{\text {sect }}\right)$ is the approximated measured OV HV BPL coupling transfer function column vector of the modified topology that comes from the application of the best L1PMA for different monotonic sections $k_{\text {sect }}$. Here, it should be noted that as the 
original OV HV BPL topology is already known via [1], so does its theoretical OV HV BPL coupling transfer function as well as its best L1PMA approximation.

Based on eq. (7) and eq. (9), FIIM can recognize either fault or instability that may occur across the transmission power grid and warn the responsible personnel. Actually, FIIM first computes the CSPpM difference between the original OV HV BPL topology and the modified one that is given by

$$
\triangle C S P p M^{*}=C S P p M-C S P p M^{*}
$$

Then, FIIM compares $\triangle \operatorname{CSPpM}^{*}$ with a warning threshold $\triangle \operatorname{CSPp}_{\mathrm{thr}}{ }^{*}$. Details concerning the determination of the warning threshold $\triangle \operatorname{CSPp}_{\mathrm{thr}}{ }^{*}$ and the relative decisions are provided in SecIVD-F.

\subsection{Traditional FIIM}

The traditional FIIM is mainly based on the observation and the experience of the responsible personnel. When greater differences of the usual case of measured OV HV coupling transfer function are observed then a warning is issued. However, the traditional FIIM is significantly unreliable due to the fact that a temporal or continuous concatenation of significant measurement differences (e.g., physical phenomena, random events) may wrongfully trigger the alarm.

In mathematical terms, traditional FIIM can be approximated by a methodology less sophisticated than the proposed FIIM one. Indeed, the percentage error sum (PES) that is given from

$$
P E S^{*} \equiv 100 \% \cdot \frac{\sum_{i=1}^{u} \frac{\left|\mathbf{H}_{\text {meas }}^{\mathrm{WtG}}\left(f_{i}\right)-\mathbf{H}_{\text {meas }}^{\mathrm{WtG}}\left(f_{i}\right)\right|}{\left|\mathbf{H}_{\text {meas }}^{\mathrm{WtG}}\left(f_{i}\right)\right|}}{u}
$$

is compared against an empirical warning threshold $P E S_{\text {thr }}{ }^{*}$ that is typically equal to zero (risky decisions). If $P E S^{*}$ is greater or equal than $P E S_{\text {thr }}{ }^{*}$, a warning message is sent to the responsible personnel. As already been mentioned, this warning threshold primarily depends on the experience of the personnel and the employee's individuality.

\section{Numerical Results and Discussion}

\subsection{Simulation Goals and Parameters}

Various types of transmission BPL networks are simulated with the purpose of evaluating the proposed FIIM against the traditional one. Similarly to the TIM of [1], the efficiency of the FIIM is assessed with regards to the transmission BPL topologies and the nature of measurement differences.

As regards the hybrid method and best L1PMA specifications, those are the same with [1]. More specifically, the BPL frequency range and the flat-fading subchannel frequency spacing are assumed equal to $1-30 \mathrm{MHz}$ and $1 \mathrm{MHz}$, respectively. Therefore, the number of subchannels $u$ in the examined frequency range is equal to 30 . Arbitrarily, the $\mathrm{WtG}^{3}$ coupling scheme is applied during the following simulations. Finally, the maximum number of monotonic sections $k_{\text {sect,max }}$ that is going to be used is assumed to be equal to 20 [37]. 


\subsection{CSPM, CSPpM and Indicative OV HV BPL Topologies}

As already been reported in [1], [37] and Sec.IIIA, there are six categories that can create significant measurement differences between measurements and theoretical results during the determination of OV HV BPL coupling transfer functions. The total occurred fault due to the six measurement difference categories, which is described in eq. (1) and eq. (8), can be assumed to follow either CUD with minimum value $-a_{\mathrm{CUD}}$ and maximum value $a_{\mathrm{CUD}}$ or normal distribution ND with mean $m_{\mathrm{ND}}$ and standard deviation $S$ ND.

With reference to eq. (7), $C S P p M$ is based on the $C S P M$ tot of TIM [1] and acts as the accompanying performance metric of FIIM. In Fig. 2(a), the CSPM tot of the indicative urban, suburban, rural and "LOS" topologies is plotted versus the CUD maximum value of the occurred measurement differences. Similar curves with Fig. 2(a) are given in Fig. 2(b) but for different values of ND standard deviation when $m_{\mathrm{ND}}$ is equal to $0 \mathrm{~dB}$. In Figs. 3(a) and 3(b), the same plots are given with Figs. 2(a) and 2(d) but for the CSPPM.

From Fig. 2(a), 2(b), 3(a) and 3(b), it is evident that:

- CSPM and CSPpM are generally increasing functions of CUD maximum value or ND standard deviation regardless of the OV HV BPL topology examined. Since both CSPM and CSPpM describe the integrity of OV HV BPL coupling transfer functions, as the measurement difference magnitudes increase so does the diversity of transfer functions.

- Although CSPM and CSPpM are both increasing functions of CUD maximum value or ND standard deviation, their behavior with respect to the multipath environment intensity changes. For given CUD maximum value or ND standard deviation, as the multipath environment becomes richer, CSPM curves increase whereas CSPpM curves decrease. Say, CSPM curves of "LOS" topology generally lay on bottom whereas the respective CSPpM ones lay on top.

- The smoothness trend of CSPM and CSPpM curves (i.e., the transition from zero to a maximum value), the maximum value of the curves and the curve notches act as an identity pattern for given OV HV BPL topology. This property of CSPM and CSPpM curves is exploited by the FIIM in order to distinguish the faults and instabilities from the measurement differences.

- The same CPSM and CSPpM conclusions can be deduced regardless of the measurement difference distribution applied (i.e, either CUD or ND).

In the following subsections, the FIIM performance is examined against the faults, instabilities and measurement differences. The proposed FIIM efficiency is compared against the traditional FIIM efficiency. Note that since the faults and instabilities that are examined in this paper has to do with branches, branch terminations and branch interconnections with the transmission lines, "LOS" topology is not further examined. Also, since the results concerning the performance of CSPM and CSPpM are almost the same and for the sake of simplicity and manuscript size, only one of the previous measurement difference distributions (e.g., CUD) will be applied, hereafter. 


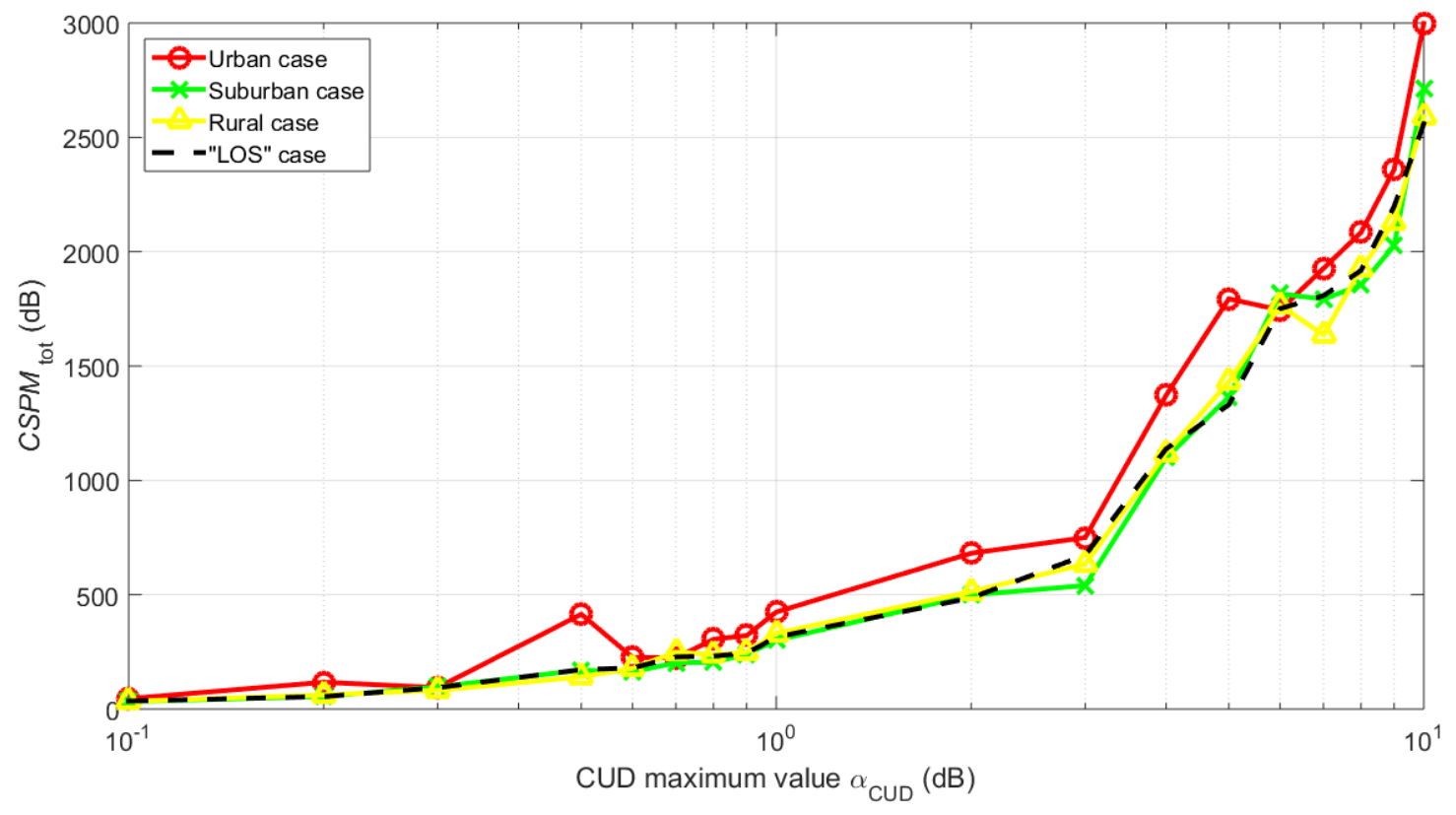

(a)

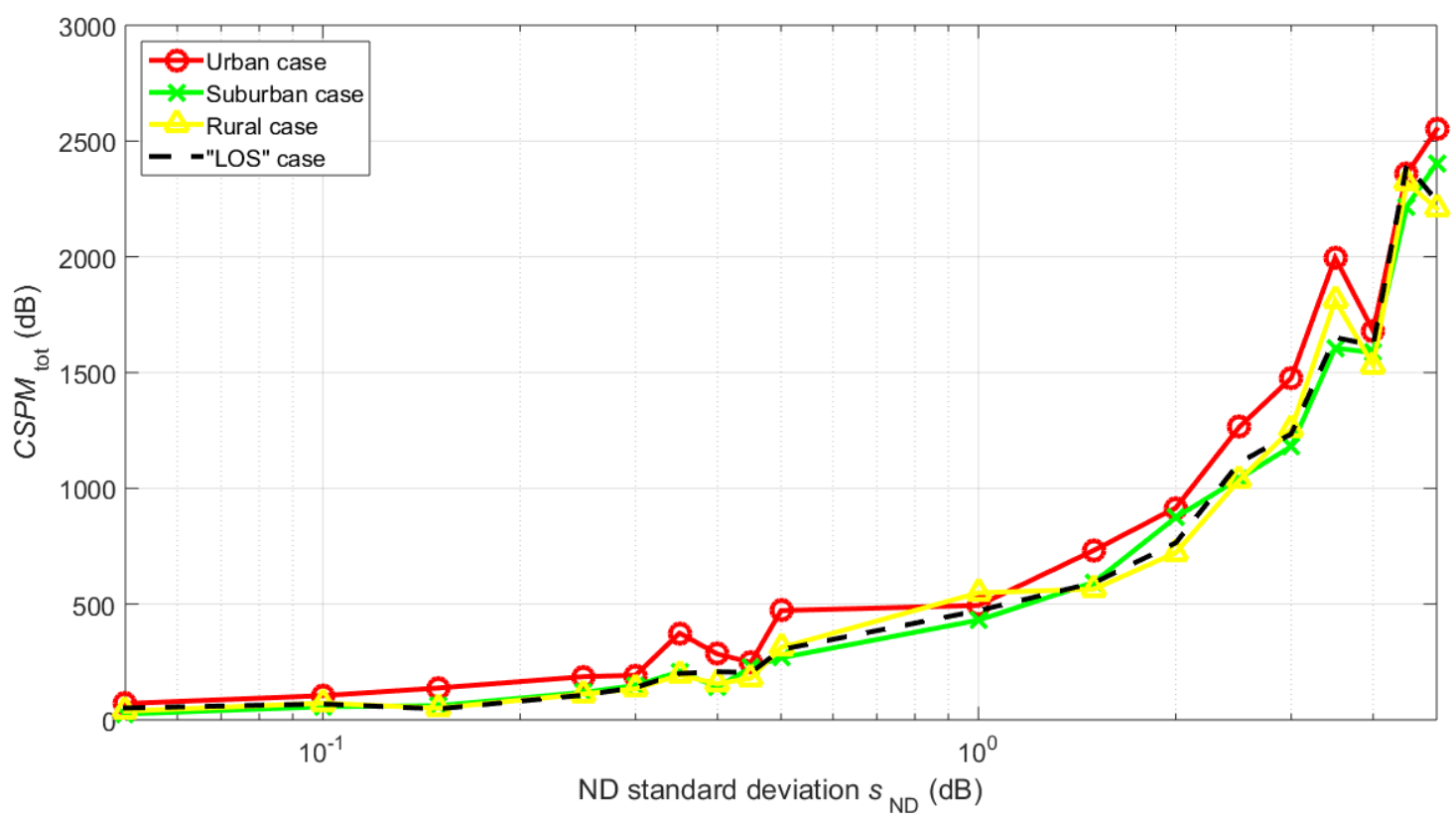

(b)

Figure 2. (a) CSPMtot versus CUD maximum value for the indicative OV HV BPL topologies. (b) CSPMtot versus ND standard deviation for the indicative OV HV BPL topologies. 


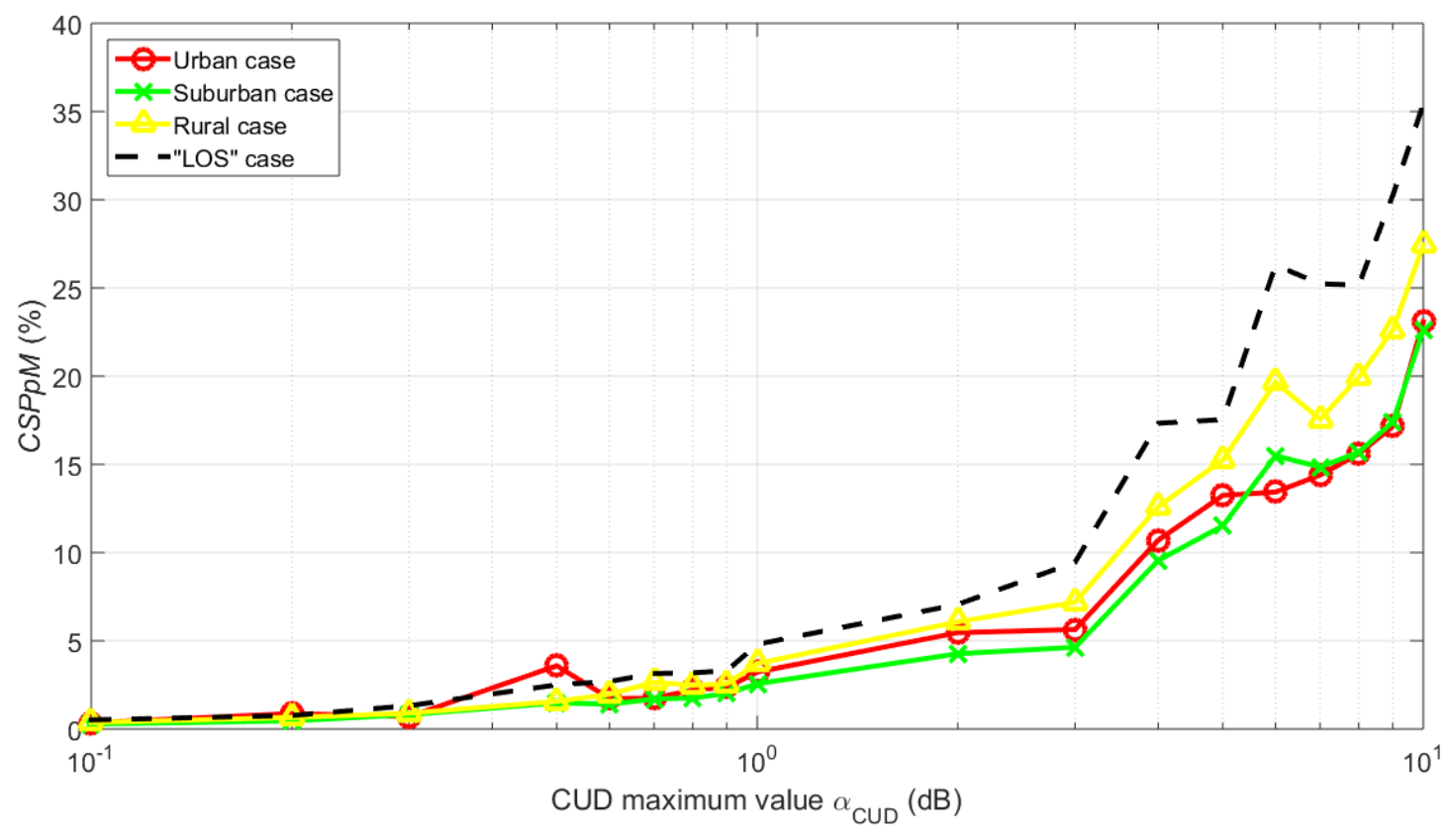

(a)

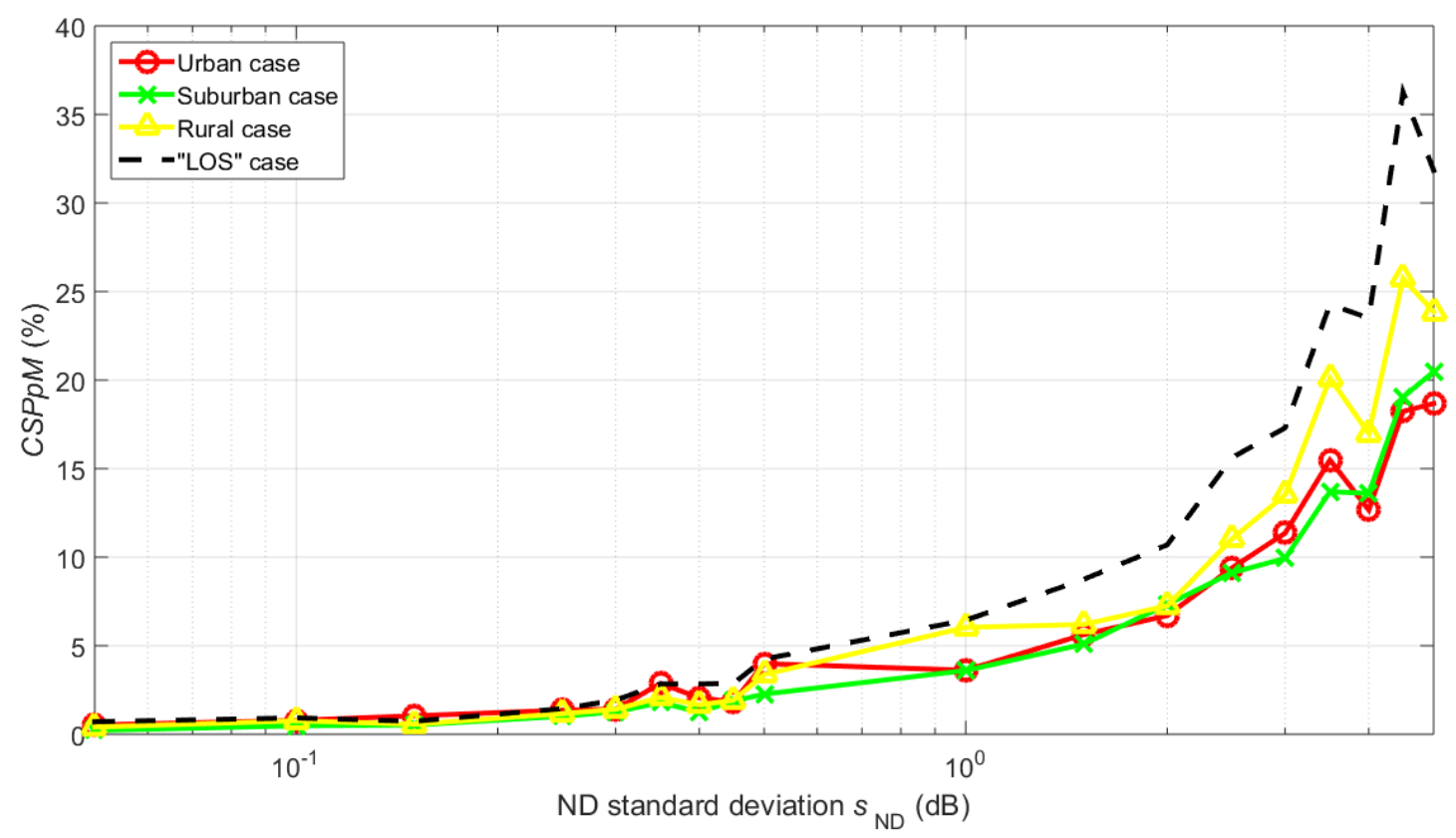

(b)

Figure 3. Same curves with Fig. 2 but for CSPpM.

\subsection{Fault in Transmission Line}

As already been reported in Sec.IIB, this fault subcategory deals with an interrupted transmission line across the transmission power grid. With reference to Fig. 1(b), since this fault has as a result the immediate communications failure between the transmitting and receiving end, its identification is a straightforward procedure. When such a case occurs, a warning is issued to the responsible personnel. Anyway, details 
concerning the exact fault position localization are outside of the scope of this paper and this problematic case is not analyzed further.

\subsection{Fault in Branch Line}

This fault subcategory describes the condition where a branch line is interrupted. With reference to Fig. 1(c), let the first branch of each indicative OV HV BPL topology be broken at $100 \mathrm{~m}$ from the branching interconnection $\mathrm{A}_{1}$ with the transmission line. Note that the interruption of the first branch, which is treated as an open circuit in this subsection, transfers the termination from $L_{\mathrm{b} 1}$ to $L_{\mathrm{b}, \mathrm{f}}=100 \mathrm{~m}$, thus, converting the examined original OV HV BPL topologies into new modified ones. The modified OV HV BPL topologies are characterized by new respective OV HV BPL coupling transfer functions and measurement differences. It is expected that the multipath environment of the modified OV HV BPL topologies becomes richer than the one of the original OV HV BPL topologies due to the new shorter branch.

Even if two different measurement differences occur during the determination of the original and modified OV HV BPL coupling transfer functions, FIIM recognizes the branch line fault and warns the responsible personnel. On the basis of eq. (10), FIIM can identify branch line faults by applying $\triangle C S P p M^{*}$. In Fig. 4(a), CSPpM of the original urban OV HV BPL topology, CSPpM ${ }^{*}$ of the modified urban OV HV BPL topology and their $\triangle C S P M^{*}$ are plotted versus the CUD maximum value of the occurred measurement differences. Note that even though two different measurement difference CUDs are applied during the determination of original and modified OV HV BPL coupling transfer functions, respectively, their CUD maximum value $\alpha_{\text {CUD }}$ remains the same for each curve marker. This is due to the fact that the surrounding environment of either the original OV HV BPL topology or its modified version remains the same and so does the chargeable event of measurement differences. Similar curves with Fig. 4(a) are given in Figs. 4(b) and 4(c) but for the suburban and rural case, respectively.

From Figs. 4(a)-(c), it is evident that CSPM"s of all the modified OV HV BPL topologies significantly differ from the respective original CSPMs. Especially, the difference, which is expressed by the respective $\triangle \mathrm{CSPpM}$ curves, is easily observable when measurement differences remain relatively low. As the measurement differences increase, the $\Delta \mathrm{CSpM}^{*}$ values decrease and so does the identification potential of branch faults.

With reference to Figs. 3(a) and 3(b), a strict $\triangle \mathrm{CSpM}^{*}$ threshold is proposed that comes from the equity of the examined $\triangle \mathrm{CSpM}^{*}$ with the CSPpM of the respective original OV HV BPL topology in order not to confuse the branch fault with the high magnitude of measurement differences. When $\triangle \operatorname{CSPPM}^{*}$ is greater or equal than $\triangle C \operatorname{SPp} M_{\mathrm{thr}}{ }^{*}$, either fault or instability surely occurs across the OV HV BPL network and a warning message must be sent to the responsible personnel. If $\triangle C S P M^{*}$ ranges from approximately $2-3 \mathrm{~dB}$ to $\triangle C S P p M_{\text {thr }}{ }^{*}$ then there is significant possibility of fault across the OV HV BPL network and a preparation of the responsible personnel should be made. 


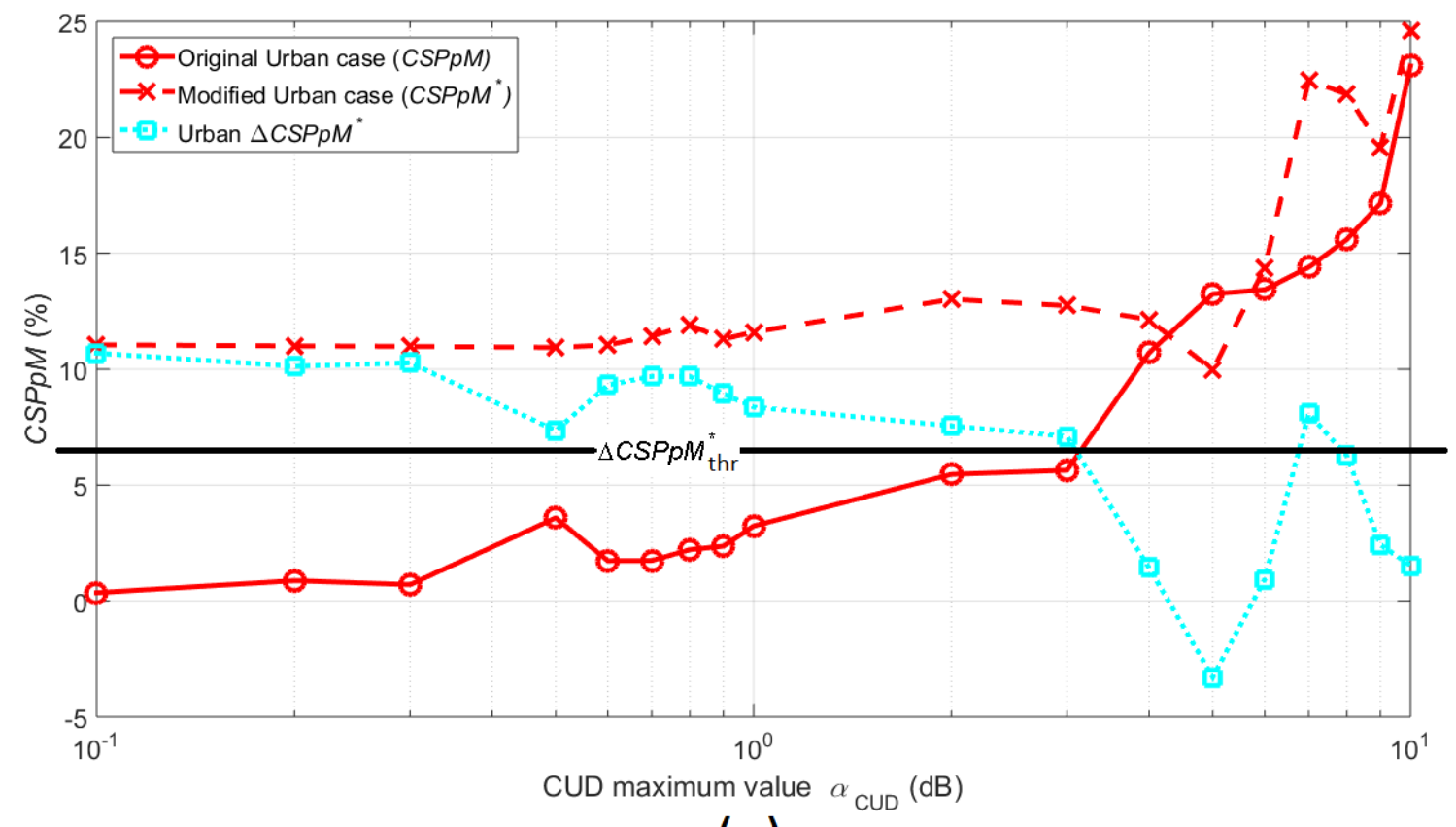

(a) 


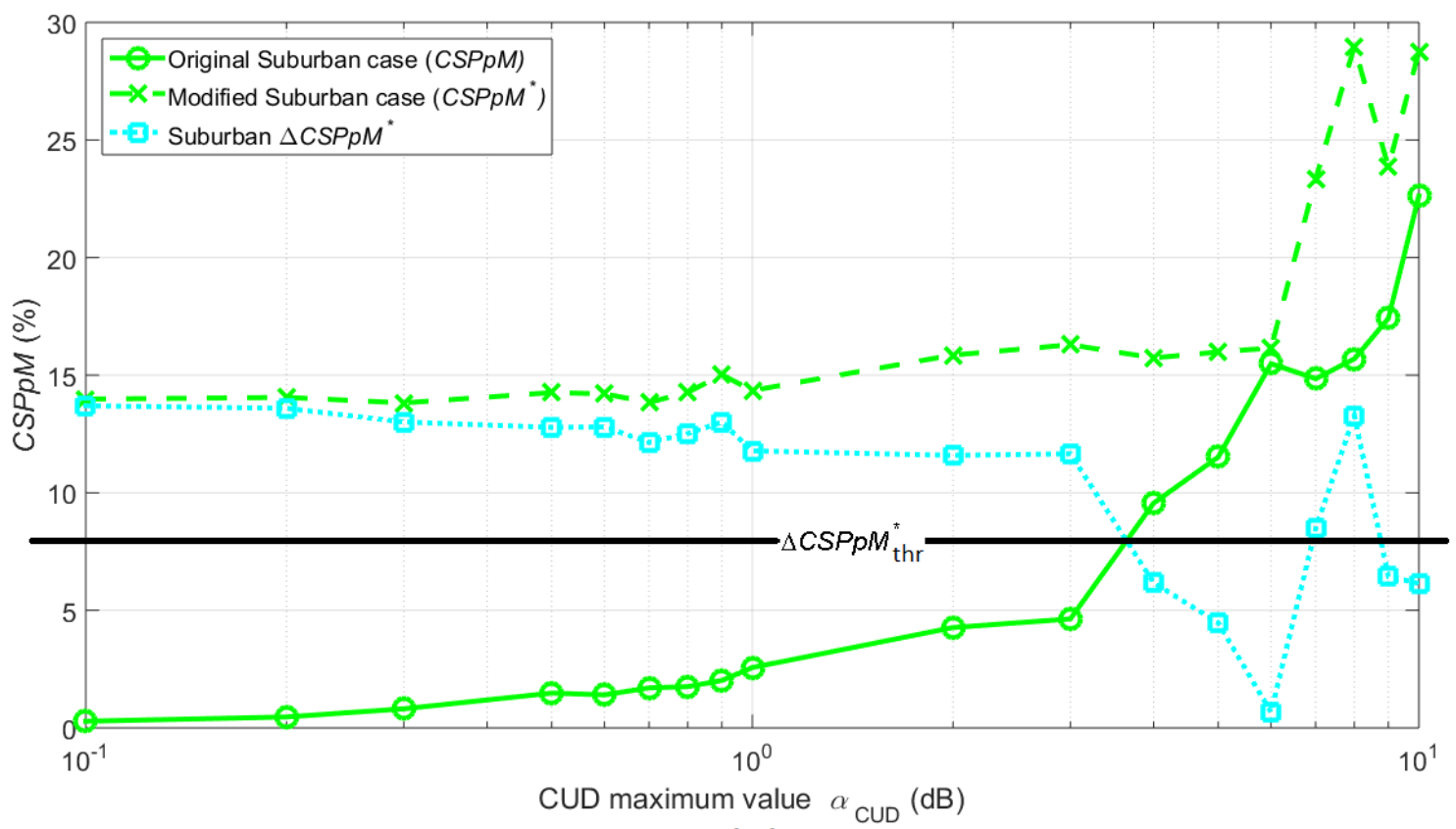

(b)

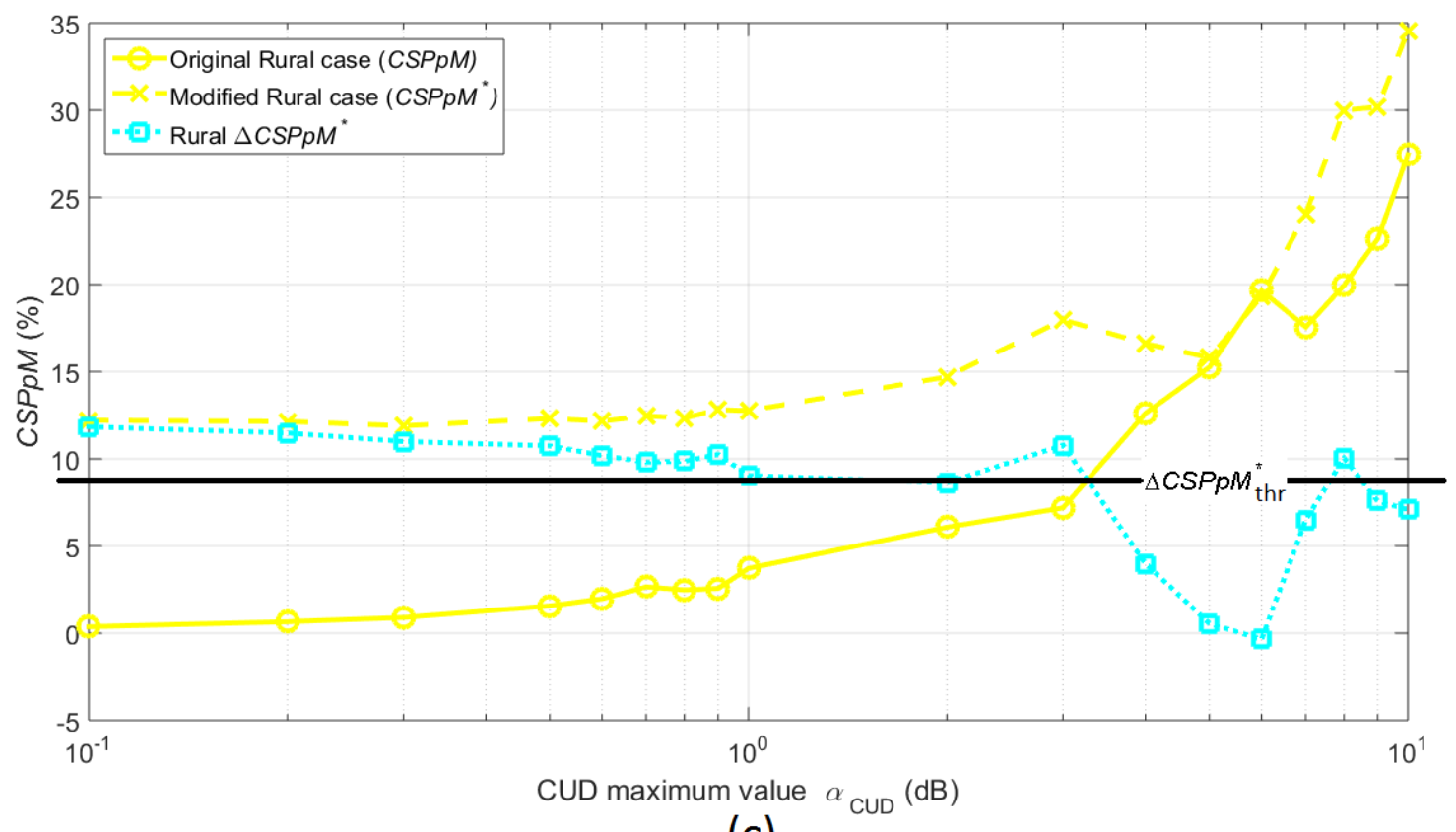

(c)

Figure 4. Fault in the first branch of the indicative OV HV BPL topologies and the behavior of

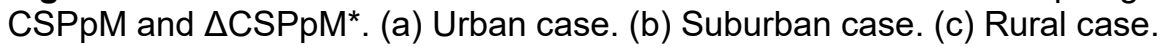

Conversely, if $\triangle C S P p M^{*}$ is below $2-3 \mathrm{~dB}$ then there is uncertainty concerning the presence of fault or instability across the OV HV BPL network. Not to trigger a false alert, $\triangle \operatorname{CSPp} M_{\text {thr }}{ }^{*}$ should receive a decent value that ranges from $6 \%$ to $9 \%$. For example, $\triangle C S P p M_{\text {thr }}{ }^{*}$ is equal to $6.44 \%, 7.97 \%$ and $8.67 \%$ for the urban, suburban and rural topology, respectively. 


\subsection{Instability in Branch Interconnection}

This subsection examines the possibility of identifying an instability that occurs in a branch interconnection. With reference to Fig. 1(d), the interruption of the last branch at the point $\mathrm{A}_{N}$ cancels the presence of this branch, thus, converting the examined $N$-branch OV HV BPL topology into a $(N-1)$-branch OV HV BPL topology. Similarly to the faults in branch lines, the modified OV HV BPL topologies are characterized by new respective OV HV BPL coupling transfer functions and measurement differences.

Based on eq. (10), FIIM can identify branch interconnection faults by applying $\triangle C S P M^{*}$. Similarly to branch line faults, in Fig. 5(a), CSPpM of the original urban OV HV BPL topology, CSPpM ${ }^{*}$ of the modified urban OV HV BPL topology and their $\triangle C S P M^{*}$ are plotted versus the CUD maximum value of the occurred measurement differences. Similar curves with Fig. 5(a) are given in Figs. 5(b) and 5(c) but for the suburban and rural case, respectively. 


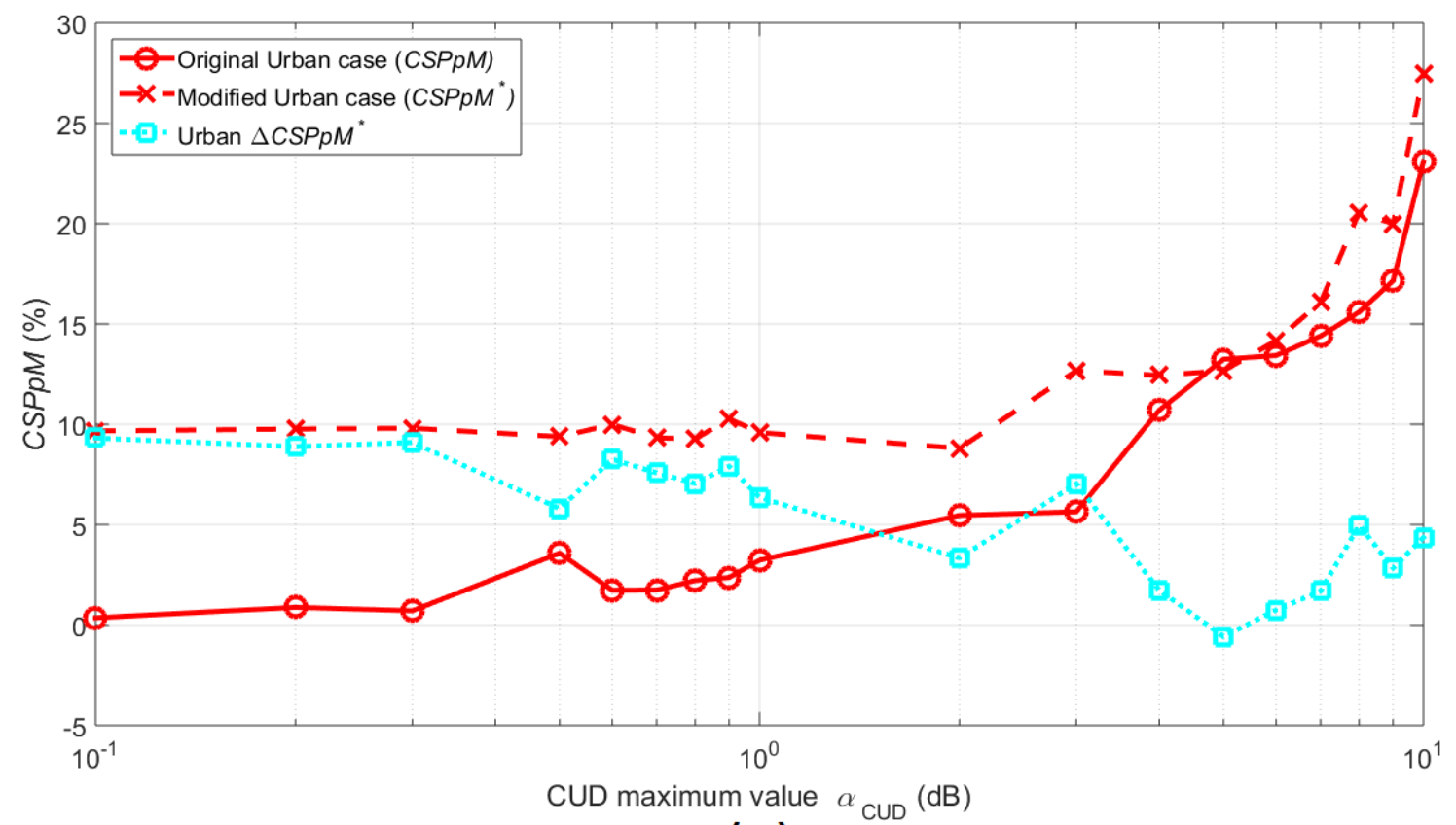

(a)

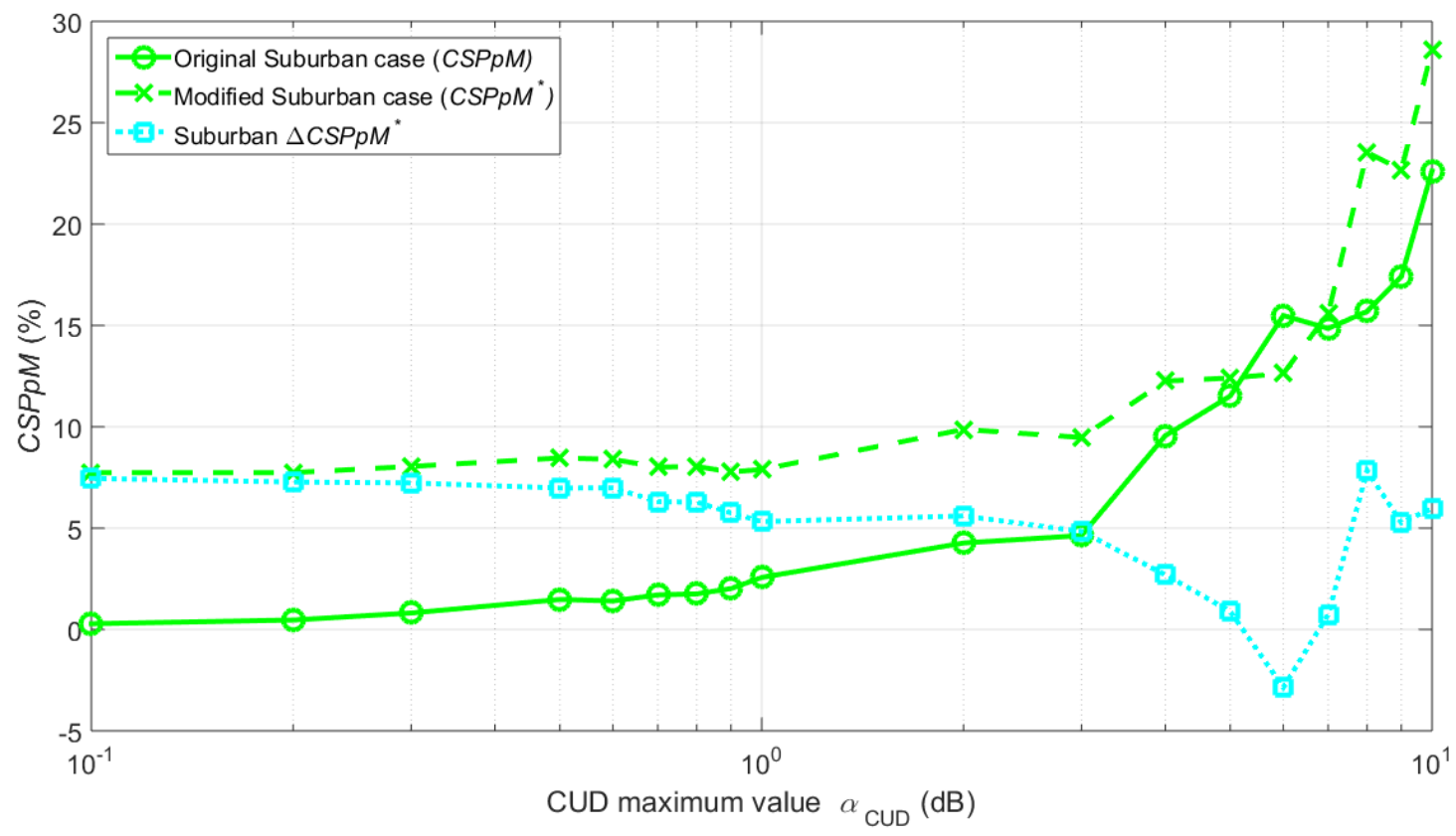

(b) 


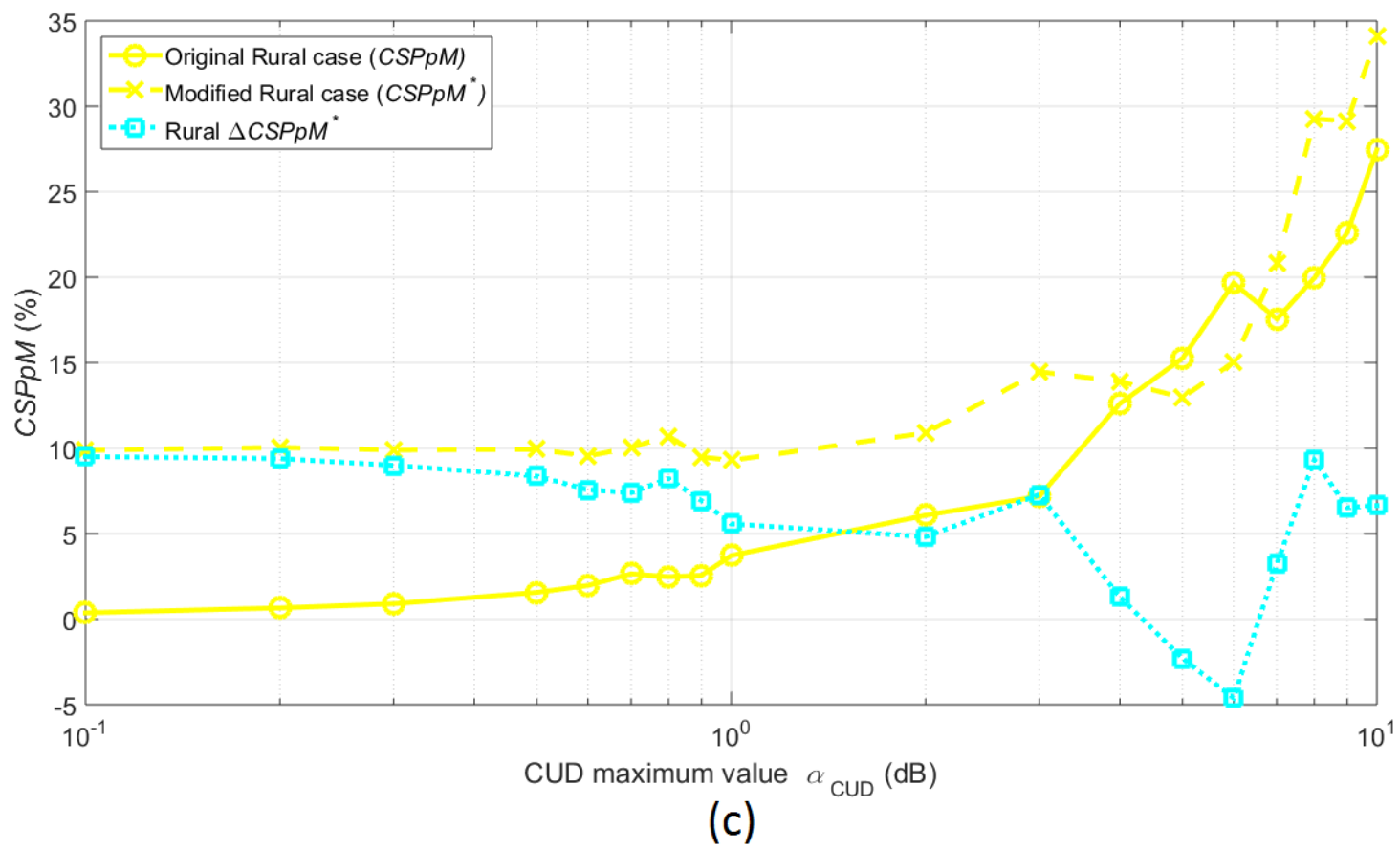

Figure 5. Instability in the last branch interconnection of the indicative OV HV BPL topologies and the behavior of CSPpM and $\triangle \mathrm{CSPpM}^{*}$. (a) Urban case. (b) Suburban case. (c) Rural case.

Comparing Figs. 5(a)-(c) with the respective Figs. 4(a)-(c), $\triangle C S P p M^{*}$ s of branch interconnection instability present lower values in comparison with the respective ones of branch line faults. This indicates that the branch interconnection instability establishes a problematic condition more difficult to be recognized in relation with the one of branch line fault. Nevertheless, $\triangle C S P p M^{*}$ identifies the branch interconnection instability regardless of the considered OV HV BPL topology and the applied CUD magnitude. Although the identification of the branch interconnection instability becomes more challenging when measurement differences magnitude exceeds $4 \mathrm{~dB}, \triangle \operatorname{CSP} M^{*}$ triggers the alarm in the majority of the cases since $\triangle C S P P M^{*}$ values exceed zero in all these cases. If a stricter decision needs to be taken then $\triangle C S P p M_{\text {thr }}{ }^{*}$ ranges from $5 \%$ to $7 \%$. The latter threshold values are lower than the ones of Sec.IVD; it is clear that the difficulty of the identification of branch interconnection instability is also reflected on $\triangle \operatorname{CSPP}_{\mathrm{thr}}{ }^{*}$.

\subsection{Instability in Branch Terminations}

This subsection examines the possibility of identifying an instability that occurs in a branch termination. Prior to proceeding to the analysis of this problematic condition, it is expected that this condition is going to be the more challenging one since FIIM needs to identify an instability that comes from a circuit anomaly at a specific piece of equipment. With reference to Fig. 1(e), let the branch termination of the first branch of each indicative OV HV BPL topology stop to act as open circuit termination. Since the branch termination fluctuates, OV HV BPL coupling transfer functions present 
instabilities that resemble to measurement differences. Assuming that the modified OV HV BPL topologies present a general short circuit in their HV/MV transformers at their first branch, FIIM can identify that an instability occurs by applying $\triangle C S P p M^{*}$; similarly to branch line faults and branch interconnection instabilities, in Fig. 6(a), CSPpM of the original urban OV HV BPL topology, $\mathrm{CSPpM}^{*}$ of the modified urban OV HV BPL topology and their $\triangle C S P p M^{*}$ are plotted versus the CUD maximum value of the occurred measurement differences. Similar curves with Fig. 6(a) are given in Figs. 6(b) and 6(c) but for the suburban and rural case, respectively.

Also, in Figs. 7(a)-(c), similar plots are curved with the respective Figs. 6(a)-(c) but for matched termination at the first branch (e.g., connection of a BPL unit at the HV/MV transformer). 


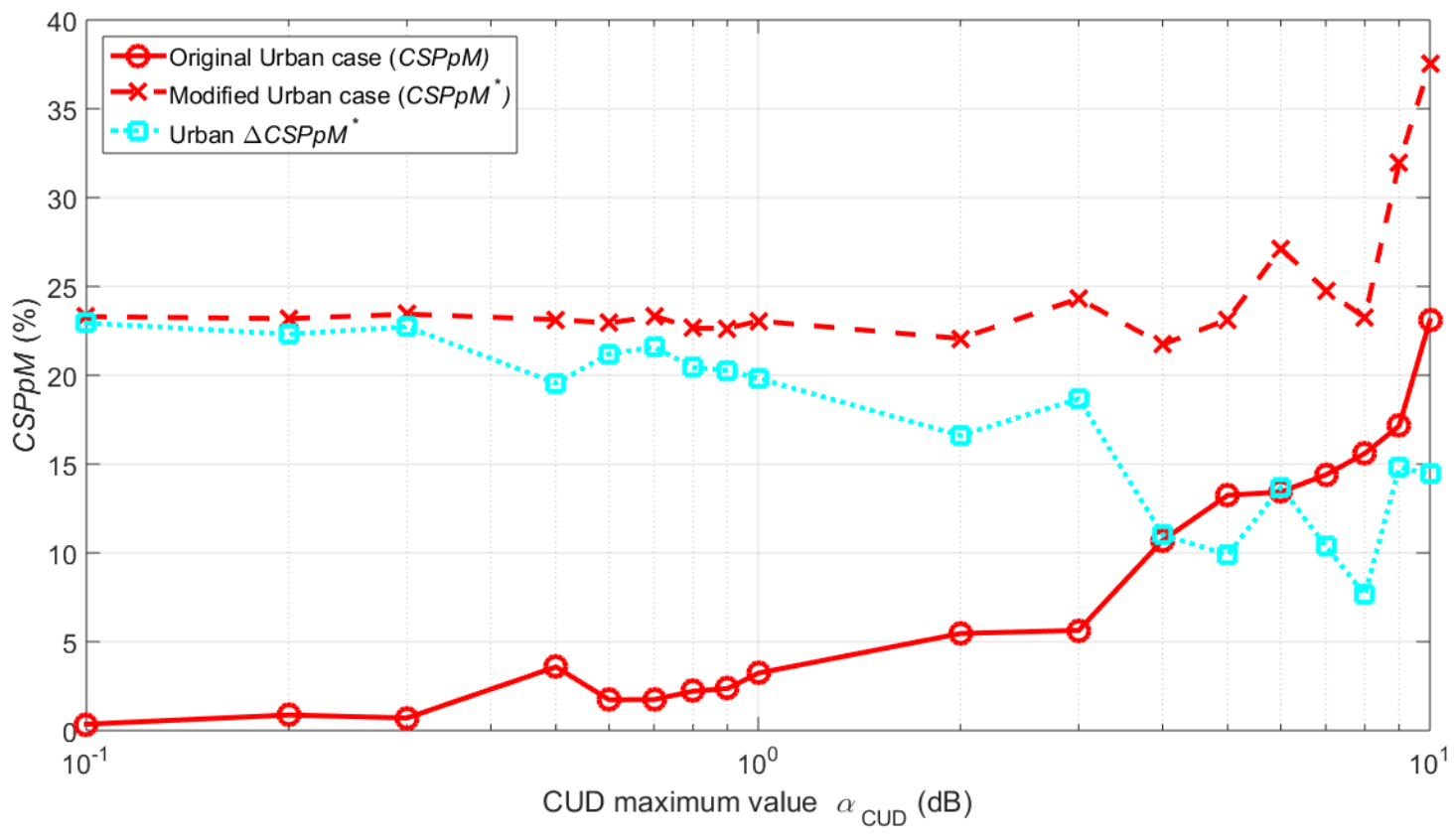

(a)

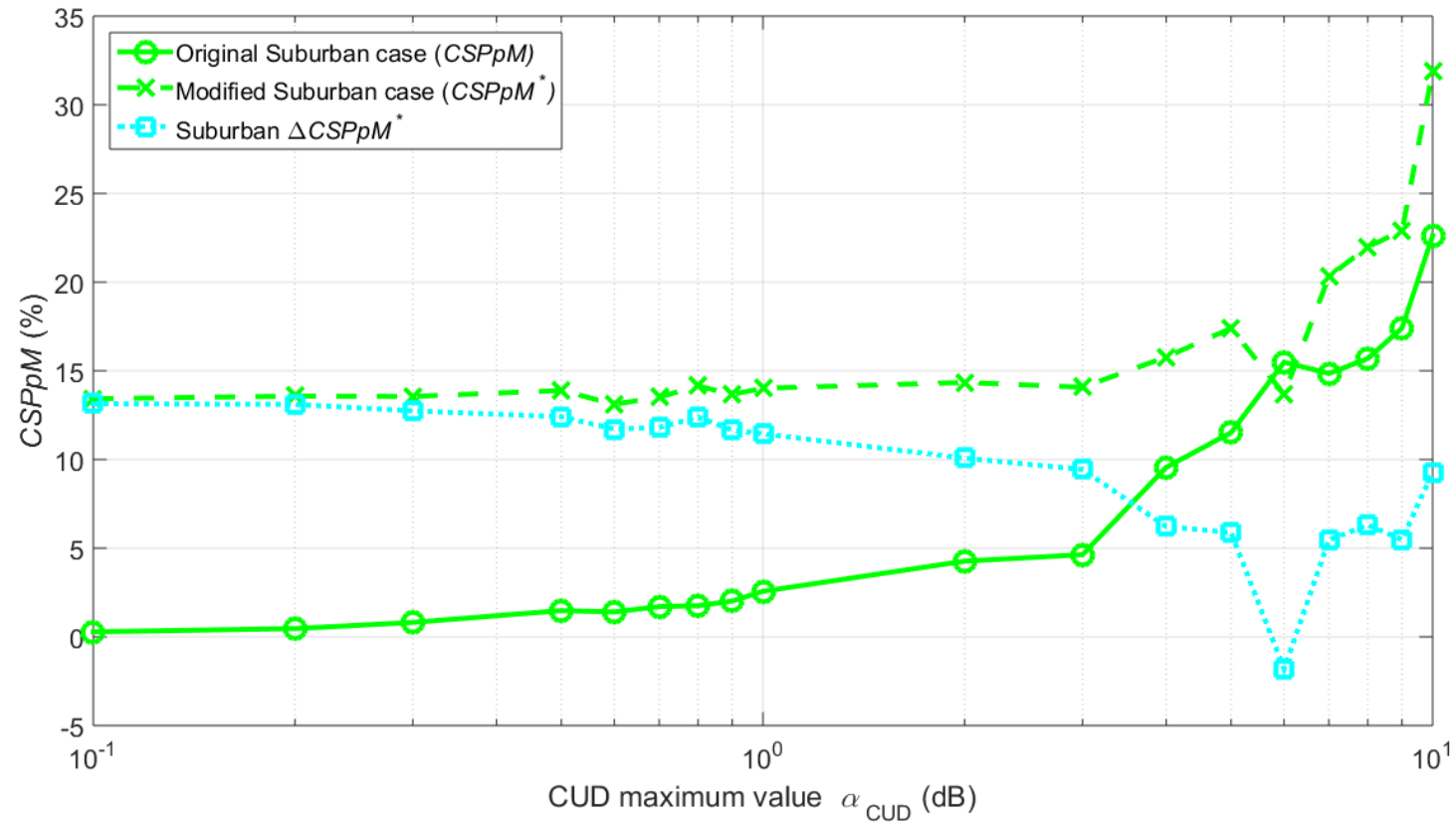

(b) 


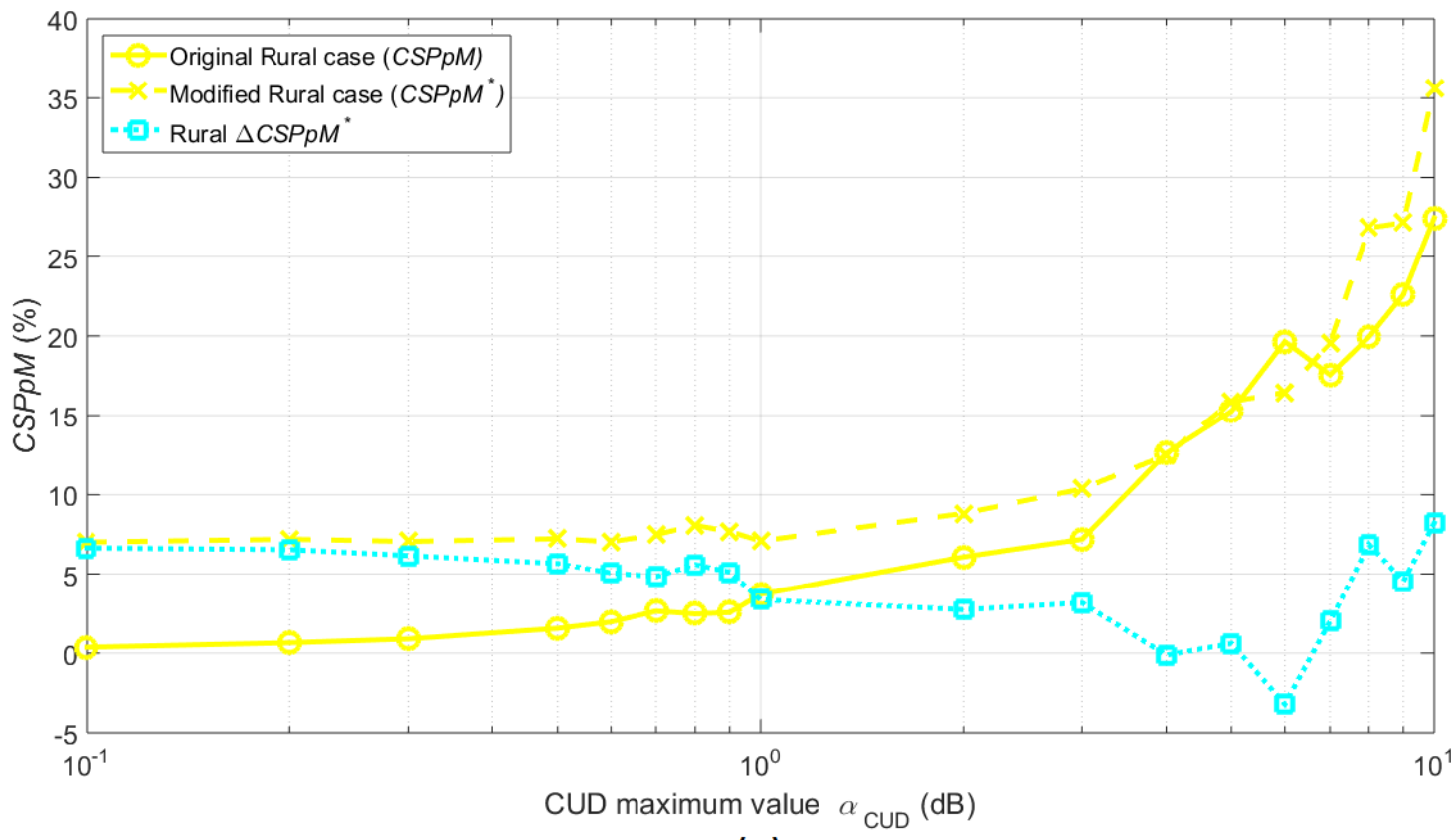

(c)

Figure 6. Termination instability (short circuit) at the first branch of the indicative OV HV BPL topologies and the behavior of CSPpM and $\triangle \mathrm{CSPpM}^{*}$. (a) Urban case. (b) Suburban case. (c) Rural case. 


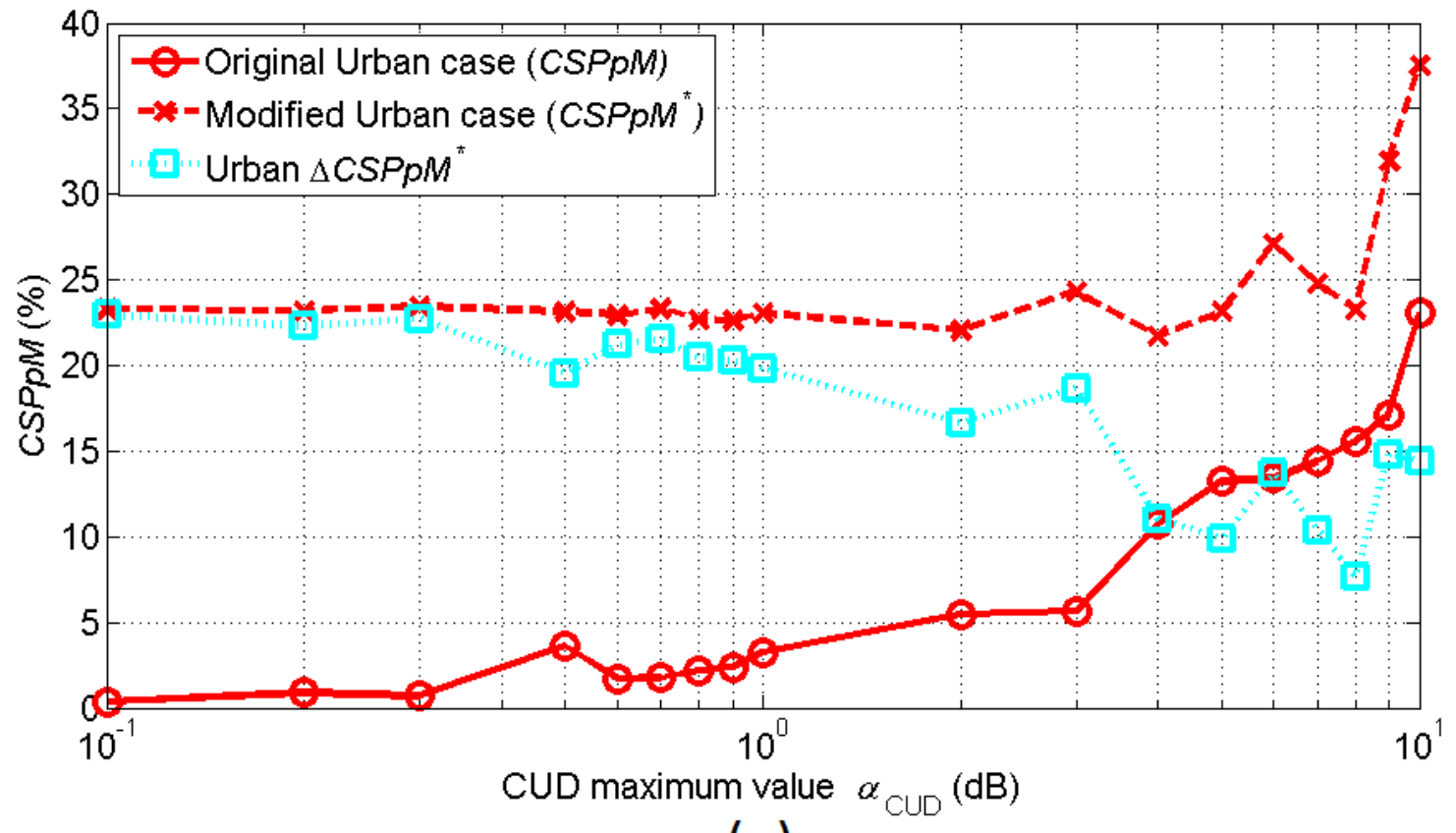

(a)

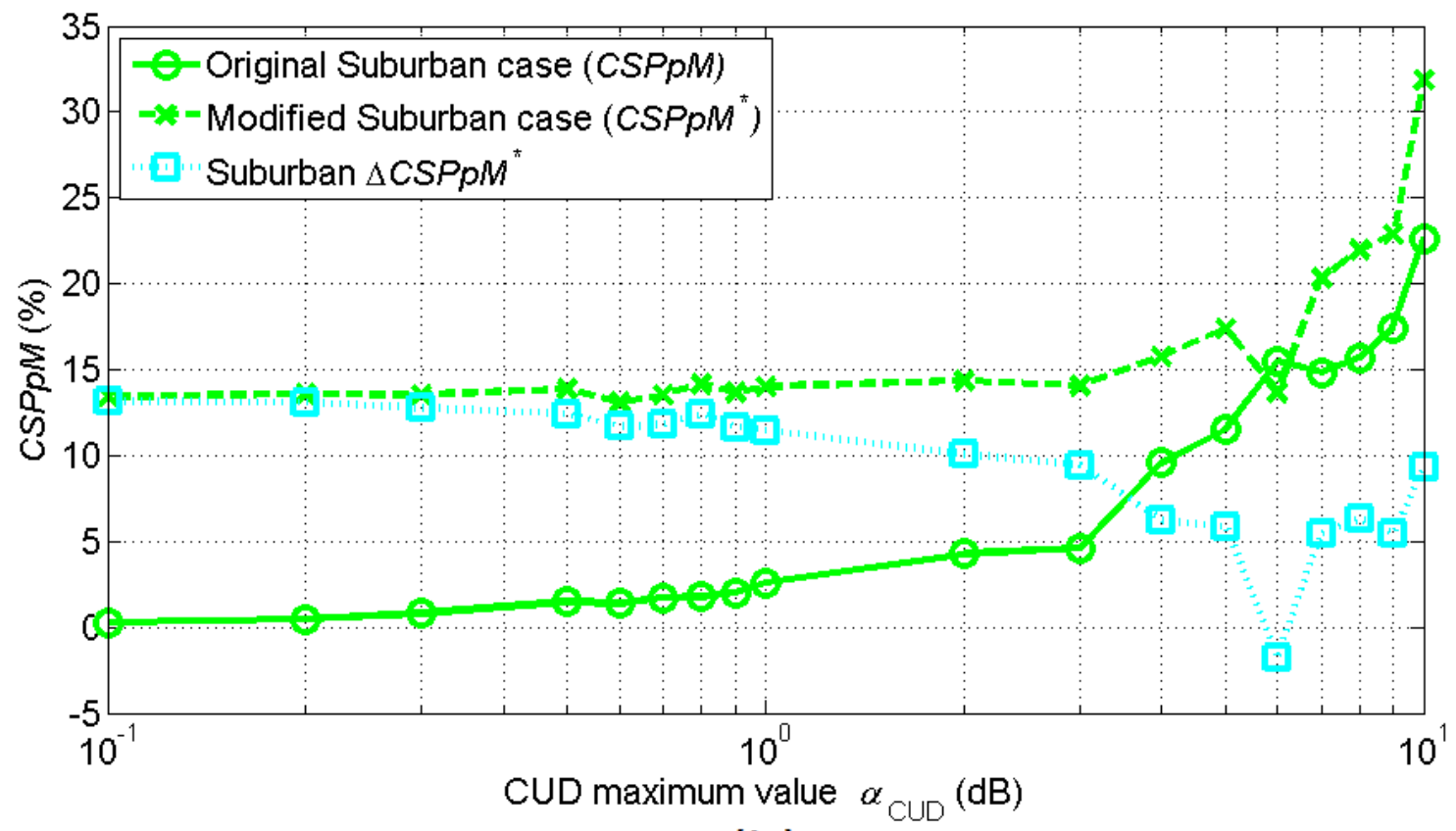

(b) 


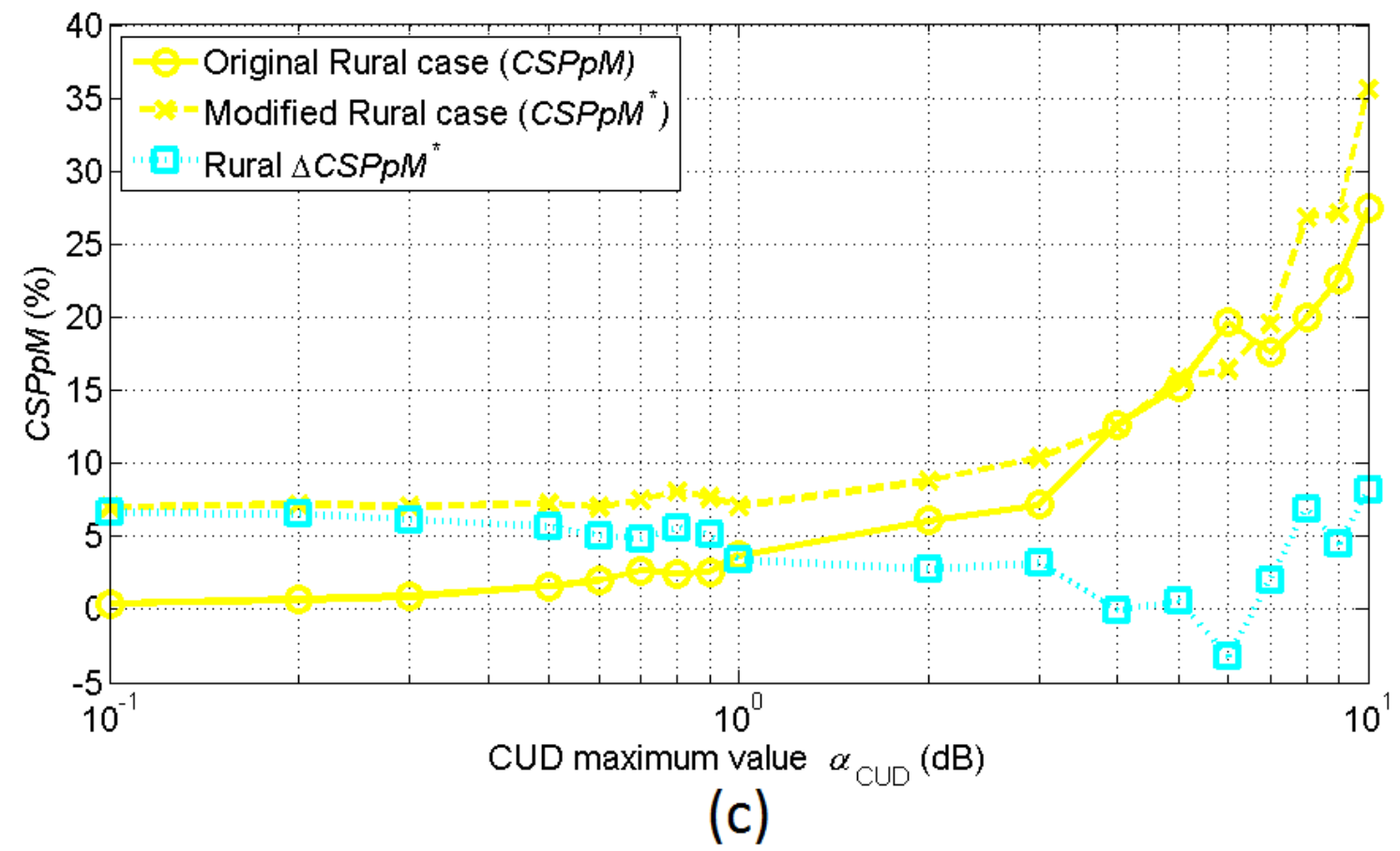

Figure 7. Same plots with Fig. 6 but for matched termination at the first branch.

From Figs. 6(a)-(c) and Figs. 7(a)-(c), FIIM can immediately identify any damage or change concerning pieces of equipment across transmission power grid. Although the termination instabilities define a rather difficult challenge, as it is seen by the lower $\triangle \operatorname{CSPP}_{\mathrm{thr}}{ }^{*} \mathrm{~s}$ in comparison with the ones of the former examined problematic conditions, FIIM can separate measurement differences from the termination instabilities. Indeed, $\triangle C S P p M^{*}$ presents positive values in the vast majority of the cases examined, even if measurement differences of $10 \mathrm{~dB}$ are present. Also, as the length of the termination branch increases, the behavior of OV HV BPL coupling transfer function tends to the one with matched termination rendering the identification more difficult. For example, CSPpM curves of the original and modified rural case little differ each other due to this power divider behavior of long branches regardless of the type of termination -see Figs. 6(c) and Fig. 7(c)-.

\subsection{Performance of Traditional FIIM}

The traditional FIIM is mainly based on the observation and the experience of the responsible personnel. With reference to eq. (11), the responsible employee should recognize unusual activity between measurements of different time instances and trigger the alarm. Each time instance is assumed to be characterized by different measurement difference CUDs but of the same maximum value since the general conditions regarding the surrounding environment do not change (same as in Secs.IVC-IVF). However, the fault and instability warning of the traditional FIIM proves to be a risky venture in all the kinds of faults and instabilities. More specifically:

- Fault in Branch Lines: Respective figures with Figs. 4(a)-(c) are given in Figs. 8(a)-(c). More analytically, in Figs. 8(a)-(c), the PES* is plotted versus the 
CUD maximum value of the occurred measurement differences for the urban, suburban and rural topology, respectively. In each figure, both the PES* of the original topology and the modified one are given when the branch line fault occurs.

- Instability in Branch Interconnections: Respective figures with Figs. 5(a)-(c) are given in Figs. 8(d)-(f). Note that Figs. 8(d)-(f) are the same figures with Figs. 8(a)-(c) but when the branch interconnection instability occurs.

- Instability in Branch Terminations: Here, two different cases are examined, as follows:

- Matched Termination: Respective figures with Figs. 6(a)-(c) are presented in Figs. 8(g)-(i). Note that Figs. 8(d)-(f) are the same figures with Figs. 8(a)-(c) but when the matched termination occurs.

- Shor Circuit Termination: Respective figures with Figs. 7(a)-(c) are demonstrated in Figs. 8(j)-(1). Note that Figs. 8(j)-(1) are the same figures with Figs. 8(a)-(c) but when the short circuit termination occurs. 


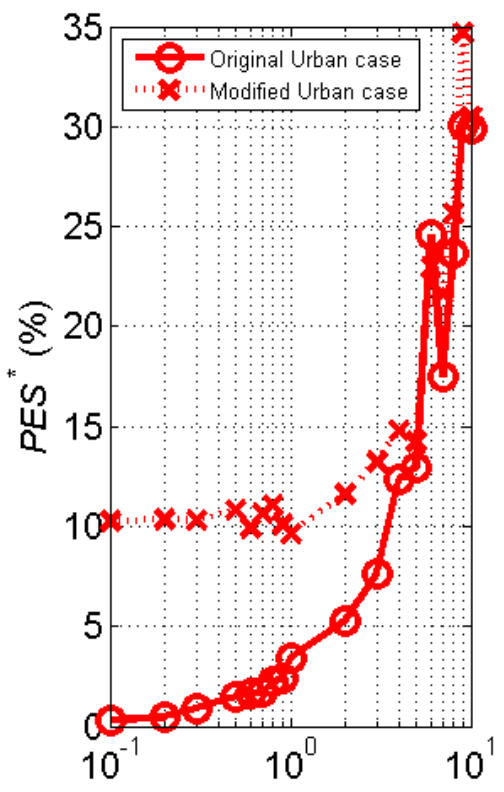

(a)

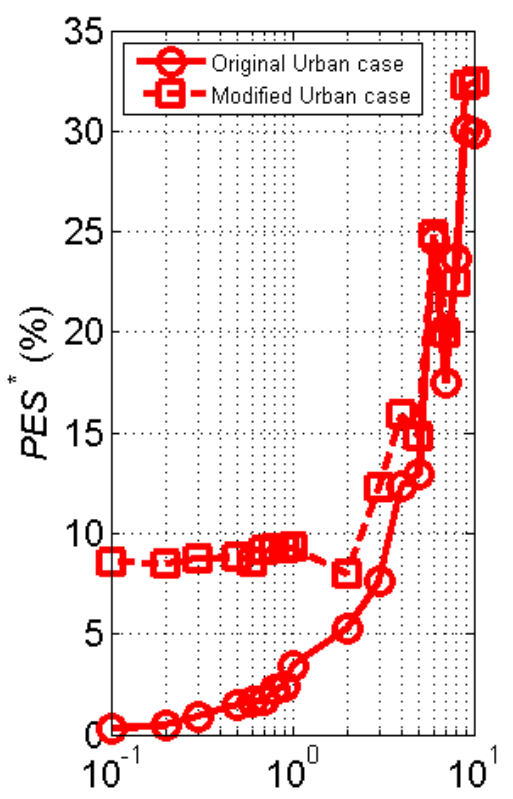

(d)

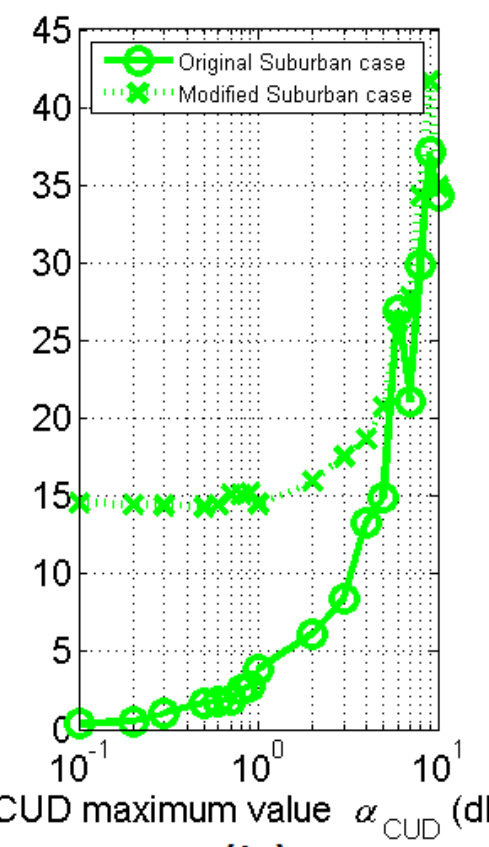

(b)

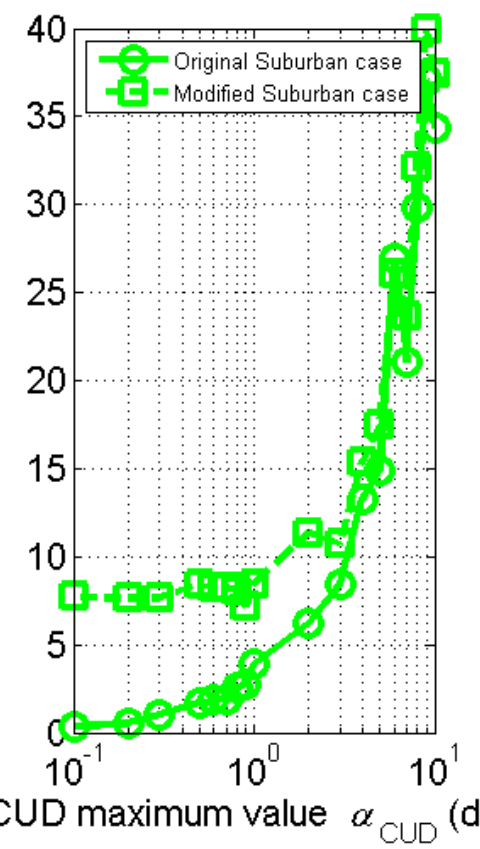

(e)

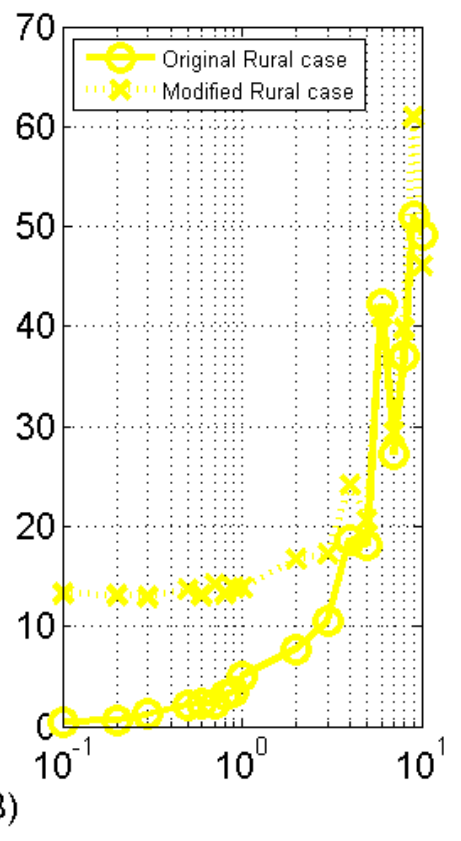

(c)

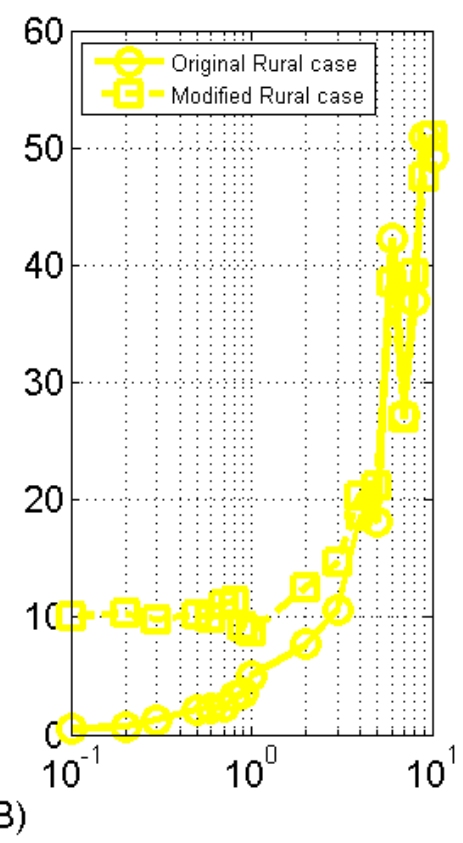

(f) 


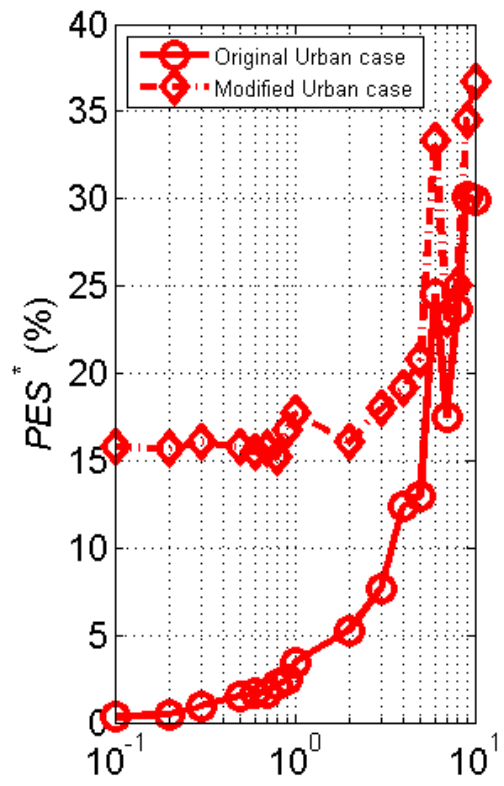

(g)

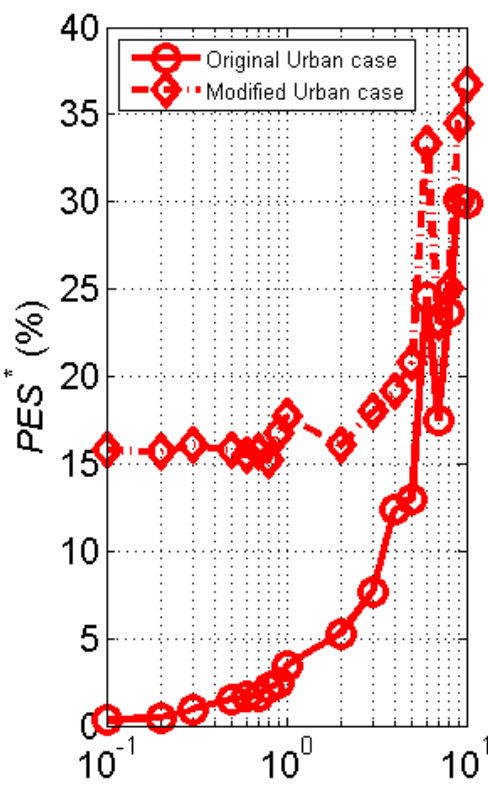

(j)

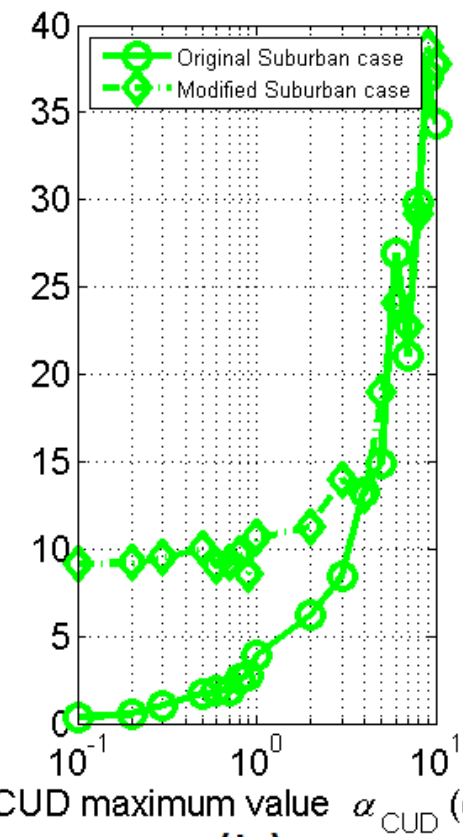

(h)

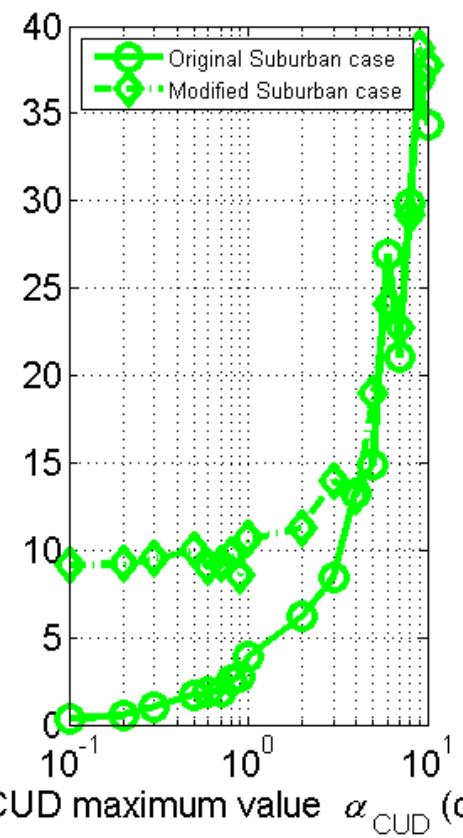

(k)

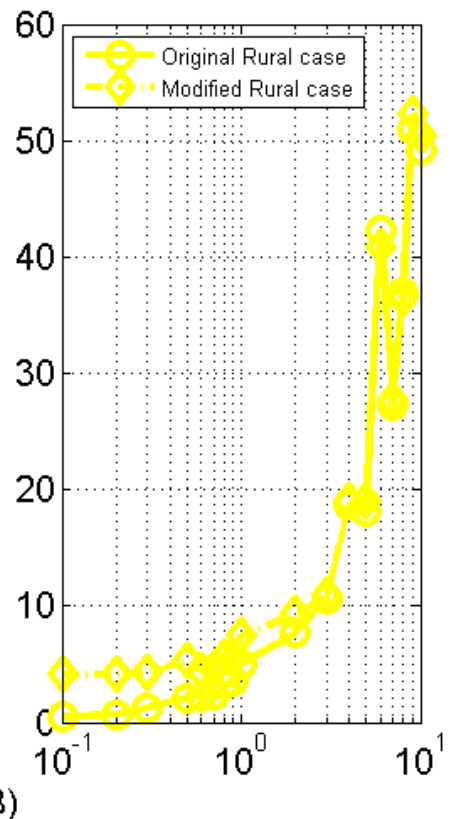

(i)

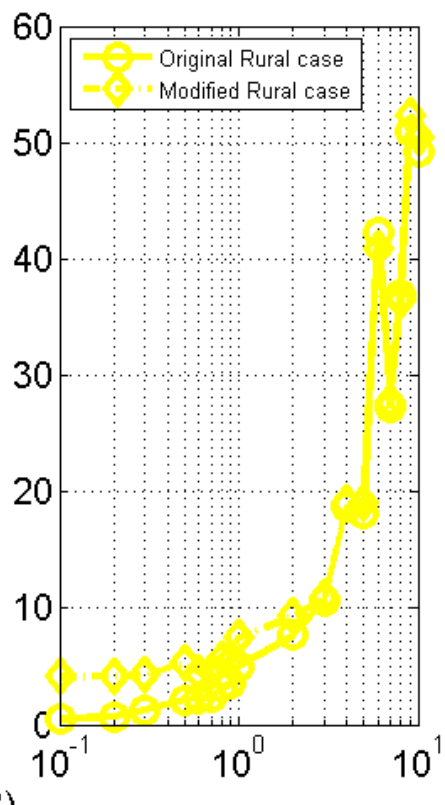

(l)

Figure 8. Traditional FIIM, faults and instabilities. (a)-(c) Branch line faults. (d)-(f) Branch interconnection instability. (g)-(i) Branch termination instability (matched termination). (j)-(I) Branch termination instability (short circuit terminations).

Comparing the performance of the proposed and traditional FIIM, it is evident that the decision regarding the various faults and instabilities that may occur across the transmission power grid is easier by applying the proposed FIIM in all the cases examined. Especially, when the measurement differences exceed $3 \mathrm{~dB}$, the proposed FIIM can still give a reliable identification regarding faults and instabilities despite the large 
measurement differences. But the main deficiency of the traditional FIIM is its performance when faults and instabilities occur in OV HV BPL topologies of poor multipath environment (e.g., indicative rural OV HV BPL topology). In these cases, the traditional FIIM faces difficulties to identify either a fault or instability since the differences between original and modified PES* curves remain marginal even if small measurement differences are applied. In fact, PES* curves are significantly affected by the presented measurement differences in contrast with the respective $\Delta \mathrm{CSPpM}^{*}$ curves that are less distorted by the measurement differences due to the error filtering of the best L1PMA. Finally, if $\mathrm{PES}^{*}$ thr is assumed to be equal to $\mathrm{CSPpM}^{*}$ thr (i.e., safe decisions are required) then traditional FIIM is treated as extremely conservative ignoring a great number of problematic conditions.

\subsection{Future Research}

From both papers, it is clearly indicated that either TIM or FIIM may be used as the basis for a plethora of smart grid applications concerning system protection, load and distributed generation management, distribution automations and diagnostic monitoring [52]. More analitically, among the following research steps, the combined operation of TIM and FIIM is going to focus on: (i) fault recognition and location; (ii) VAR control; (iii) isolation of faults on single phases; (iv) predictive maintenance; and (v) EMI and noise analysis.

To implement the aforementioned research goals, some structural changes should be made either in TIM or in FIIM procedure. As the TIM is concerned, the OV HV BPL topology database should be enriched so that higher accuracy is achieved (i.e., lower values for the length spacing, higher values for the maximum branch length and a variety of branch terminations) and more multi-branch OV HV BPL topologies are inserted. This implies that significant work should be done in the field of the optimization of the database (i.e., faster algorithms during the database creation, insertion and update). As the FIIM is concerned, CSPpM curves should be further analyzed in order to become identity metrics for the OV HV BPL topologies and $\triangle \mathrm{CSPpM}^{*}$ curves should be further investigated in order to become fault identities for various faults and instabilities that may occur across the transmission power grid. Finally, to become real-time application, significant optimization efforts should be concentrated to the minimization of FIIM computations and the reduction of today's overall execution times of FIIM for the various fault and instability conditions.

\section{Conclusions}

In this companion paper, the proposed TIM of [1] has extended to FIIM that deals with the fault and instability identification across transmission power grids. FIIM consists of: (i) the hybrid method; (ii) the best L1PMA; (iii) TIM; and (iv) CSPpM and $\triangle \mathrm{CSPpM}^{*}$ that serve as fault identification metrics.

As the assessment of the FIIM is concerned, FIIM has easily achieved to identify faults in branch lines, instabilities in branch interconnections and instabilities in branch terminations of various kinds during the real-time operation of indicative OV HV BPL topologies. Actually, FIIM have identified the faults and instabilities in all the cases examined even though measurement differences up to $10 \mathrm{~dB}$ have been considered.

Exploiting the virtues of the emerging intelligent energy systems, both TIM and FIIM can be an invaluable tool during the surveillance and monitoring of transmission and distribution power grid supporting a myriad of smart grid applications. 


\section{Conflicts of Interest}

The author declares that there is no conflict of interests regarding the publication of this paper.

\section{References}

[1] A. G. Lazaropoulos, "Measurement Differences, Faults and Instabilities in Intelligent Energy Systems - Part 1: Identification of Overhead High-Voltage Broadband over Power Lines Network Topologies by Applying Topology Identification Methodology (TIM)," Trends in Renewable Energy, vol. 2, no. 3, pp. 85-112, 2016. DOI: 10.17737/tre.2016.2.3.0026

[2] A. Milioudis, G. T. Andreou, and D. P. Labridis, "Detection and location of high impedance faults in multiconductor overhead distribution lines using power line communication devices," IEEE Trans. on Smart Grid, vol. 6, no. 2, pp. 894-902, 2015.

[3] T. A. Papadopoulos, A. I. Chrysochos, E. O. Kontis, and G. K. Papagiannis, "Ringdown Analysis of Power Systems Using Vector Fitting," Electric Power Systems Research, vol. 141, pp. 100-103, 2016.

[4] A. Milioudis, G. Andreou, and D. Labridis, "Optimum transmitted power spectral distribution for broadband power line communication systems considering electromagnetic emissions," Elsevier Electric Power Systems Research, in press, 2016.

[5] S. S. Pappas, L. Ekonomou, D. C. Karamousantas, G. E. Chatzarakis, S. K. Katsikas, and P. Liatsis, "Electricity Demand Loads Modeling Using AutoRegressive Moving Average (ARMA) Models," Energy, vol. 33, no. 9, pp. 1353-1360, 2008. DOI: 10.1016/j.energy.2008.05.008

[6] R. Lee and R. H. Osborn, "A microcomputer based data acquisition system for high impedance fault analysis," IEEE Power Eng. Rev., vol. PER-5, no. 10, p. 35, Oct. 1985.

[7] S. Ebron, D. Lubkeman, and M. White, "A neural network approach to the detection of incipient faults on power distribution feeders," IEEE Trans. Power Del., vol. 5, no. 2, pp. 905-914, Apr. 1990.

[8] J.-H. Ko, J.-C. Shim, C.-W. Ryu, C.-G. Park, and W.-Y. Yim, Detection of high impedance faults using neural nets and chaotic degree," in Proc. 1998 Int. Conf. Energy Manage. Power Del. (EMPD), vol. 2. Singapore, pp. 399-404.

[9] F. Jota and P. R. S. Jota, "High-impedance fault identification using a fuzzy reasoning system," IEE Proc. Gener. Transmiss. Distrib., vol. 145, no. 6, pp. 656-661, Nov. 1998.

[10] Y. Sheng and S. Rovnyak, "Decision tree-based methodology for high impedance fault detection," IEEE Trans. Power Del., vol. 19, no. 2, pp. 533-536, Apr. 2004.

[11] D. C. T. Wai and X. Yibin, "A novel technique for high impedance fault identification," IEEE Trans. Power Del., vol. 13, no. 3, pp. 738-744, Jul. 1998.

[12] I. Zamora, A. Mazon, K. J. Sagastabeitia, and J. Zamora, "New method for detecting low current faults in electrical distribution systems," IEEE Trans. Power Del., vol. 22, no. 4, pp. 2072-2079, Oct. 2007.

[13] J. Zamora, I. Zamora, A. Mazon, and K. J. Sagastabeitia, "Optimal frequency value to detect low current faults superposing voltage tones," IEEE Trans. Power Del., vol. 23, no. 4, pp. 1773-1779, Oct. 2008. 
[14] M. Michalik, W. Rebizant, M. Lukowicz, S.-J. Lee, and S.-H. Kang, "Highimpedance fault detection in distribution networks with use of wavelet-based algorithm," IEEE Trans. Power Del., vol. 21, no. 4, pp. 1793-1802, Oct. 2006.

[15] M. Michalik, M. Lukowicz, W. Rebizant, S.-J. Lee, and S.-H. Kang, "Verification of the wavelet-based HIF detecting algorithm performance in solidly grounded MV networks," IEEE Trans. Power Del., vol. 22, no. 4, pp. 2057-2064, Oct. 2007.

[16] B. Aucoin and B. Russell, "Distribution high impedance fault detection utilizing high frequency current components," IEEE Trans. Power App. Syst., vol. PAS101, no. 6, pp. 1596-1606, Jun. 1982.

[17] A. N. Milioudis, G. T. Andreou, and D. P. Labridis, "Enhanced Protection Scheme for Smart Grids Using Power Line Communications Techniques-Part II: Location of High Impedance Fault Position," IEEE Trans. on Smart Grid, no. 3, vol. 4, pp. 1631-1640, 2012.

[18] A. Milioudis, G. Andreou, and D. Labridis, "High impedance fault detection using power line communication techniques," in Proc. 2010 45 th Int. Univ. Power Eng. Conf. (UPEC), Cardiff, U.K., pp. 1-6.

[19] A. Milioudis, G. Andreou, and D. Labridis, "High impedance fault evaluation using narrowband power line communication techniques," in Proc. 2011 IEEE Trondheim PowerTech, Trondheim, Norway, pp. 1-6.

[20] A. Milioudis, G. Andreou, and D. Labridis, "Enhanced protection scheme for smart grids using power line communications techniques-Part I: Detection of high impedance fault occurrence," IEEE Trans. Smart Grid, vol. 3, no. 4, pp. 1621-1630, Dec. 2012.

[21] A. I. Chrysochos, T. A. Papadopoulos, A. ElSamadouny, G. K. Papagiannis, and N. Al-Dhahir, "Optimized MIMO-OFDM design for narrowband-PLC applications in medium-voltage smart distribution grids," Electric Power Systems Research, vol. 140, pp. 253-262, 2016. DOI: 10.1016/j.epsr.2016.06.017

[22] A. G. Lazaropoulos, "Factors Influencing Broadband Transmission Characteristics of Underground Low-Voltage Distribution Networks," IET Commun., vol. 6, no. 17, pp. 2886-2893, Nov. 2012.

[23] A. G. Lazaropoulos and P. G. Cottis, "Transmission characteristics of overhead medium voltage power line communication channels," IEEE Trans. Power Del., vol. 24, no. 3, pp. 1164-1173, Jul. 2009.

[24] A. G. Lazaropoulos and P. G. Cottis, "Capacity of overhead medium voltage power line communication channels," IEEE Trans. Power Del., vol. 25, no. 2, pp. 723-733, Apr. 2010.

[25] A. G. Lazaropoulos and P. G. Cottis, "Broadband transmission via underground medium-voltage power lines-Part I: transmission characteristics," IEEE Trans. Power Del., vol. 25, no. 4, pp. 2414-2424, Oct. 2010.

[26] A. G. Lazaropoulos and P. G. Cottis, "Broadband transmission via underground medium-voltage power lines-Part II: capacity," IEEE Trans. Power Del., vol. 25, no. 4, pp. 2425-2434, Oct. 2010.

[27] A. G. Lazaropoulos, "Broadband transmission characteristics of overhead highvoltage power line communication channels," Progress in Electromagnetics Research B, vol. 36, pp. 373-398, 2012. [Online]. Available: http://www.jpier.org/PIERB/pierb36/19.11091408.pdf

[28] A. G. Lazaropoulos, "Towards broadband over power lines systems integration: Transmission characteristics of underground low-voltage distribution power lines," 
Progress in Electromagnetics Research B, 39, pp. 89-114, 2012. [Online]. Available: http://www.jpier.org/PIERB/pierb39/05.12012409.pdf

[29] A. G. Lazaropoulos, "Broadband transmission and statistical performance properties of overhead high-voltage transmission networks," Hindawi Journal of Computer Networks and Commun., 2012, article ID 875632, 2012. [Online]. Available: http://www.hindawi.com/journals/jcnc/aip/875632/

[30] A. G. Lazaropoulos, "Towards modal integration of overhead and underground low-voltage and medium-voltage power line communication channels in the smart grid landscape: model expansion, broadband signal transmission characteristics, and statistical performance metrics (Invited Paper)," ISRN Signal Processing, in press, [Online]. Available: http://www.isrn.com/journals/sp/aip/121628/

[31] A. G. Lazaropoulos, "Review and Progress towards the Common Broadband Management of High-Voltage Transmission Grids: Model Expansion and Comparative Modal Analysis," ISRN Electronics, vol. 2012, Article ID 935286, pp. $1-18,2012 . \quad$ [Online]. Available: http://www.hindawi.com/isrn/electronics/2012/935286/

[32] A. G. Lazaropoulos, "Review and Progress towards the Capacity Boost of Overhead and Underground Medium-Voltage and Low-Voltage Broadband over Power Lines Networks: Cooperative Communications through Two- and ThreeHop Repeater Systems," ISRN Electronics, vol. 2013, Article ID 472190, pp. 1-19, 2013. [Online]. Available: http://www.hindawi.com/isrn/electronics/aip/472190/

[33] A. G. Lazaropoulos, "Green Overhead and Underground Multiple-Input MultipleOutput Medium Voltage Broadband over Power Lines Networks: EnergyEfficient Power Control," Springer Journal of Global Optimization, vol. 2012 / Print ISSN 0925-5001, pp. 1-28, Oct. 2012.

[34] P. Amirshahi and M. Kavehrad, "High-frequency characteristics of overhead multiconductor power lines for broadband communications," IEEE J. Sel. Areas Commun., vol. 24, no. 7, pp. 1292-1303, Jul. 2006.

[35] T. Sartenaer, "Multiuser communications over frequency selective wired channels and applications to the powerline access network" Ph.D. dissertation, Univ. Catholique Louvain, Louvain-la-Neuve, Belgium, Sep. 2004.

[36] T. Calliacoudas and F. Issa, "“'Multiconductor transmission lines and cables solver," An efficient simulation tool for plc channel networks development," presented at the IEEE Int. Conf. Power Line Communications and Its Applications, Athens, Greece, Mar. 2002.

[37] A. G. Lazaropoulos, "Best L1 Piecewise Monotonic Data Approximation in Overhead and Underground Medium-Voltage and Low-Voltage Broadband over Power Lines Networks: Theoretical and Practical Transfer Function Determination," Hindawi Journal of Computational Engineering, vol. 2016, Article ID 6762390, 24 pages, 2016. doi: 10.1155/2016/6762390.

[38] I. C. Demetriou and M. J. D. Powell, "Least squares smoothing of univariate data to achieve piecewise monotonicity," IMA J. of Numerical Analysis, vol. 11, pp. 411-432, 1991.

[39] I. C. Demetriou and V. Koutoulidis"On Signal Restoration by Piecewise Monotonic Approximation", in Lecture Notes in Engineering and Computer Science: Proceedings of The World Congress on Engineering 2013,London, U.K., Jul. 2013, pp. 268-273. 
[40] I. C. Demetriou, "An application of best $L 1$ piecewise monotonic data approximation to signal restoration," IAENG International Journal of Applied Mathematics, vol. 53, no. 4, pp. 226-232, 2013.

[41] I. C. Demetriou, "L1PMA: A Fortran 77 Package for Best L1 Piecewise Monotonic Data Smoothing," Computer Physics Communications, vol. 151, no. 1, pp. 315-338, 2003.

[42] I. C. Demetriou, "Data Smoothing by Piecewise Monotonic Divided Differences," Ph.D. Dissertation, Department of Applied Mathematics and Theoretical Physics, University of Cambridge, Cambridge, 1985.

[43] I. C. Demetriou, "Best L1 Piecewise Monotonic Data Modelling,"Int. Trans. Opl Res., vol. 1, no. 1, pp. 85-94,1994.

[44] I.C. Demetriou, "L1PMA: a Fortran 77 package for best L1 piecewise monotonic data smoothing," 2003 http://cpc.cs.qub.ac.uk/summaries/ADRF

[45] T. Sartenaer and P. Delogne, "Deterministic modelling of the (Shielded) outdoor powerline channel based on the multiconductor transmission line equations," IEEE J. Sel. Areas Commun., vol. 24, no. 7, pp. 1277-1291, Jul. 2006.

[46] OPERA1, D5: Pathloss as a function of frequency, distance and network topology for various LV and MV European powerline networks. IST Integrated Project No 507667, Apr. 2005.

[47] P. Amirshahi, "Broadband access and home networking through powerline networks" Ph.D. dissertation, Pennsylvania State Univ., University Park, PA, May 2006. [Online]. Available: http://etda.libraries.psu.edu/theses/approved/WorldWideIndex/ETD1205/index.html

[48] OPERA1, D44: Report presenting the architecture of plc system, the electricity network topologies, the operating modes and the equipment over which PLC access system will be installed, IST Integr. Project No 507667, Dec. 2005.

[49] T. Banwell and S. Galli, "A novel approach to accurate modeling of the indoor power line channel-Part I: Circuit analysis and companion model," IEEE Trans. Power Del., vol. 20, no. 2, pp. 655-663, Apr. 2005.

[50] S. Galli and T. Banwell, "A novel approach to accurate modeling of the indoor power line channel - Part II: Transfer function and channel properties," IEEE Trans. Power Del., vol. 20, no. 3, pp. 1869-1878, Jul. 2005.

[51] S. Galli and T. Banwell, "A deterministic frequency-domain model for the indoor power line transfer function," IEEE J. Sel. Areas Commun., vol. 24, no. 7, pp. 1304-1316, Jul. 2006.

[52] A. G. Lazaropoulos, A. M. Sarafi, and P. G. Cottis, "The emerging smart grid A pilot MV/BPL network installed at Lavrion, Greece," in Proc. Workshop on Applications for Powerline Communications WSPLC 2008, Thessaloniki, Greece, Oct. 2008.2 [Online]. Available: http://newton.ee.auth.gr/WSPLC08/Abstracts\%5CSG 3.pdf

Article copyright: (C) 2016 Athanasios G. Lazaropoulos. This is an open access article distributed under the terms of the Creative Commons Attribution 4.0 International License, which permits unrestricted use and distribution provided the original author and source are credited. 